\title{
Associative learning drives longitudinally-graded presynaptic plasticity of neurotransmitter release along axonal compartments
}

Aaron Stahl ${ }^{1}$, Nathaniel C. Noyes ${ }^{1}$, Tamara Boto1, Miao Jing², Jianzhi Zeng ${ }^{3,4,5}$, Lanikea B. King ${ }^{1}$, Yulong Li ${ }^{2,3,4,5}$, Ronald L. Davis ${ }^{1}$, Seth M. Tomchik ${ }^{1 *}$

${ }^{1}$ Department of Neuroscience, The Scripps Research Institute, Scripps Florida, Jupiter, FL 33458, USA

${ }^{2}$ Chinese Institute for Brain Research, ${ }^{3}$ State Key Laboratory of Membrane Biology, Peking University School of Life Sciences, ${ }^{4}$ Peking-Tsinghua Center for Life Sciences, ${ }^{5} \mathrm{PKU}$ IDG/McGovern Institute for Brain Research, Beijing, China

*stomchik@scripps.edu

130 Scripps Way \#3C1

Jupiter, FL 33458

(561) 228-3496

Keywords: synaptic, bidirectional, potentiation, depression, Cav2, Cacophony, IP3R 


\section{$1 \quad$ Abstract}

2 Anatomical and physiological compartmentalization of neurons is a mechanism to increase the

3 computational capacity of a circuit, and a major question is what role axonal

4 compartmentalization plays. Axonal compartmentalization may enable localized, presynaptic

5 plasticity to alter neuronal output in a flexible, experience-dependent manner. Here we show

6 that olfactory learning generates compartmentalized, bidirectional plasticity of acetylcholine

7 release that varies across the longitudinal compartments of Drosophila mushroom body (MB)

8 axons. The directionality of the learning-induced plasticity depends on the valence of the

9 learning event (aversive vs. appetitive), varies linearly across proximal to distal compartments

10 following appetitive conditioning, and correlates with learning-induced changes in downstream

11 mushroom body output neurons (MBONs) that modulate behavioral action selection.

12 Potentiation of acetylcholine release was dependent on the Cav2.1 calcium channel subunit

13 cacophony. In addition, contrast between the positive conditioned stimulus and other odors

14 required the inositol triphosphate receptor $\left(\mathrm{IP}_{3} \mathrm{R}\right)$, which was required to maintain responsivity to

15 odors in untrained conditions. Downstream from the mushroom body, a set of MBONs that

16 receive their input from the $\gamma 3 \mathrm{MB}$ compartment were required for normal appetitive learning,

17 suggesting that they represent a key node through which discriminative effects influence

18 appetitive memory and decision-making. These data demonstrate that learning drives valence-

19 correlated, compartmentalized, bidirectional potentiation and depression of synaptic

20 neurotransmitter release, which rely on distinct mechanisms and are distributed across axonal

21 compartments in a learning circuit. 


\section{Introduction}

24 Neuronal dendrites carry out computations through compartmentalized signaling, while axons

25 have long been considered to carry signals to their terminal fields relatively uniformly following

26 spike initiation. However, anatomical and physiological compartmentalization of axons has

27 been recently documented in neurons from worms through mammals (Boto et al., 2014; Cohn et

28 al., 2015; Hendricks et al., 2012; Rowan et al., 2016). How axonal compartmentalization

29 influences information flow across neuronal circuits and modulates behavioral outcomes is not

30 understood. One functional role for axonal compartmentalization may be to enable localized,

31 presynaptic plasticity to alter output from select axon compartments in a flexible, experience-

32 dependent manner. This would vastly enhance the neuron's flexibility and computational

33 capabilities. One potential function of such compartmentalization would allow independent

34 modulation of axonal segments and/or synaptic release sites by biologically-salient events, such as sensory stimuli that drive learning.

37 The anatomical organization of the Drosophila mushroom body (MB) makes it an exemplary testbed to study how sensory information is processed during learning and rerouted to alter behavioral outcomes. The MB encodes odor in sparse representations across intrinsic MB neurons, which are arranged in several parallel sets. They project axons in fasciculated

41 bundles into several anatomically-distinct, but spatially adjacent lobes $\left(\alpha / \beta, \alpha^{\prime} / \beta^{\prime}\right.$, and $\left.\gamma\right)$

42 (Crittenden et al., 1998). These bundled axons are longitudinally subdivided into discrete tiled

43 compartments (Aso et al., 2014a). Each compartment receives afferent neuromodulatory input

44 from unique dopaminergic neurons (Aso et al., 2014a; Mao and Davis, 2009), and innervates

45 unique efferent mushroom body output neurons (MBONs) (Aso et al., 2014a). Each set of

46 dopaminergic neurons plays an individual role in learning, with some conveying aversive 
47 teaching signals (Schroll et al., 2006; Schwaerzel et al., 2003), others conveying positive

48 teaching signals (Liu et al., 2012; Yamagata et al., 2015), and a third class modulating memory

49 strength without driving valence (Boto et al., 2019). Likewise, each MBON has a unique effect

50 on behavioral approach and avoidance, with some biasing the animal to approach, others

51 biasing the animal to avoidance, and some having no effect (Aso et al., 2014b; Perisse et al.,

52 2016; Placais et al., 2013; Sejourne et al., 2011).

54 A major question in learning and memory is how presynaptic plasticity contributes to reweight

55 the flow of sensory signals down each of the downstream "approach" or "avoidance" circuits,

56 altering action selection and memory retrieval. In naïve conditions, Drosophila dopaminergic

57 circuits modulate cAMP in a compartmentalized fashion along the MB axons (Boto et al., 2014).

58 This compartmentalized dopaminergic signaling can independently modulate $\mathrm{Ca}^{2+}$ responses in

59 each compartment, as well as the responses of the downstream valence-coding MBONs (Cohn

60 et al., 2015). Dopamine-dependent heterosynaptic depression at the MB-MBON synapse

61 modulates learning (Hige et al., 2015a). Therefore, presynaptic plasticity in the MB neurons

62 within each compartment could theoretically drive the changes in MBON responsiveness that

63 guide behavioral learning (Zhang et al., 2019). However, manipulation of the "aversive"

64 protocerebral posterior lateral 1 (PPL1) dopaminergic neurons does not detectably alter $\mathrm{Ca}^{2+}$

65 signals in MB neurons (Boto et al., 2019; Hige et al., 2015a). Furthermore, $\mathrm{Ca}^{2+}$ responses in

$66 \mathrm{MB}$ neurons are uniformly potentiated across compartments with appetitive classical

67 conditioning protocols and unaltered in MB neurons following aversive protocols (Louis et al.,

68 2018). This raises the question of how local, compartmentalized synaptic plasticity in MB

69 neurons drives coherent changes in downstream MBONs to modulate action selection during

70 memory retrieval. Learning/dopamine-induced plasticity has been demonstrated in the

71 downstream MBONs (Berry et al., 2018; Hige et al., 2015a; Hige et al., 2015b; Owald et al., 
72 2015), with dopamine also acting directly on MBONs (Takemura et al., 2017). Feedforward

73 inhibition among MBONs that drive opposing behavioral outcomes provides a mechanism

74 explaining how valence coding in MBONs could be generated (Perisse et al., 2016). Yet this

75 does not explain the compartmentalized, dopamine-dependent plasticity in MB neurons

76 themselves or the necessity for dopamine receptors and downstream signaling molecules in the

77 intrinsic MB neurons (Kim et al., 2007; McGuire et al., 2003; Zars et al., 2000).

79 Here we describe how learning alters the flow of information through the MB via synaptic

80 release of the putative MB neurotransmitter (Barnstedt et al., 2016), using genetically-encoded

81 indicators of synaptic acetylcholine neurotransmission. The data reveal that learning alters the

82 compartmentalized axonal acetylcholine release from Drosophila mushroom body (MB) neurons

83 in valence-specific spatiotemporal patterns, via distinct molecular mechanisms, driving

84 behavioral alterations via modulation of specific downstream output neurons.

\section{$\underline{\text { Results }}$}

Associative learning modulates neurotransmitter release in a spatially-distinct manner across longitudinal axonal compartments

89 Synapses within each MB compartment transmit olfactory information from MB neurons to 90 compartment-specific MBONs (Fig. 1A, 5A,B) (Aso et al., 2014a; Tanaka et al., 2008). The

91 MBONs exert distinct and often-opposing effects on behavior, with some innately promoting 92 approach and others promoting avoidance (Aso et al., 2014b; Berry et al., 2018; Ichinose et al., 93 2015; Owald et al., 2015; Perisse et al., 2016; Placais et al., 2013; Sayin et al., 2019; Sejourne 94 et al., 2011). Synaptic depression has been observed in the MB-MBON synapses following 

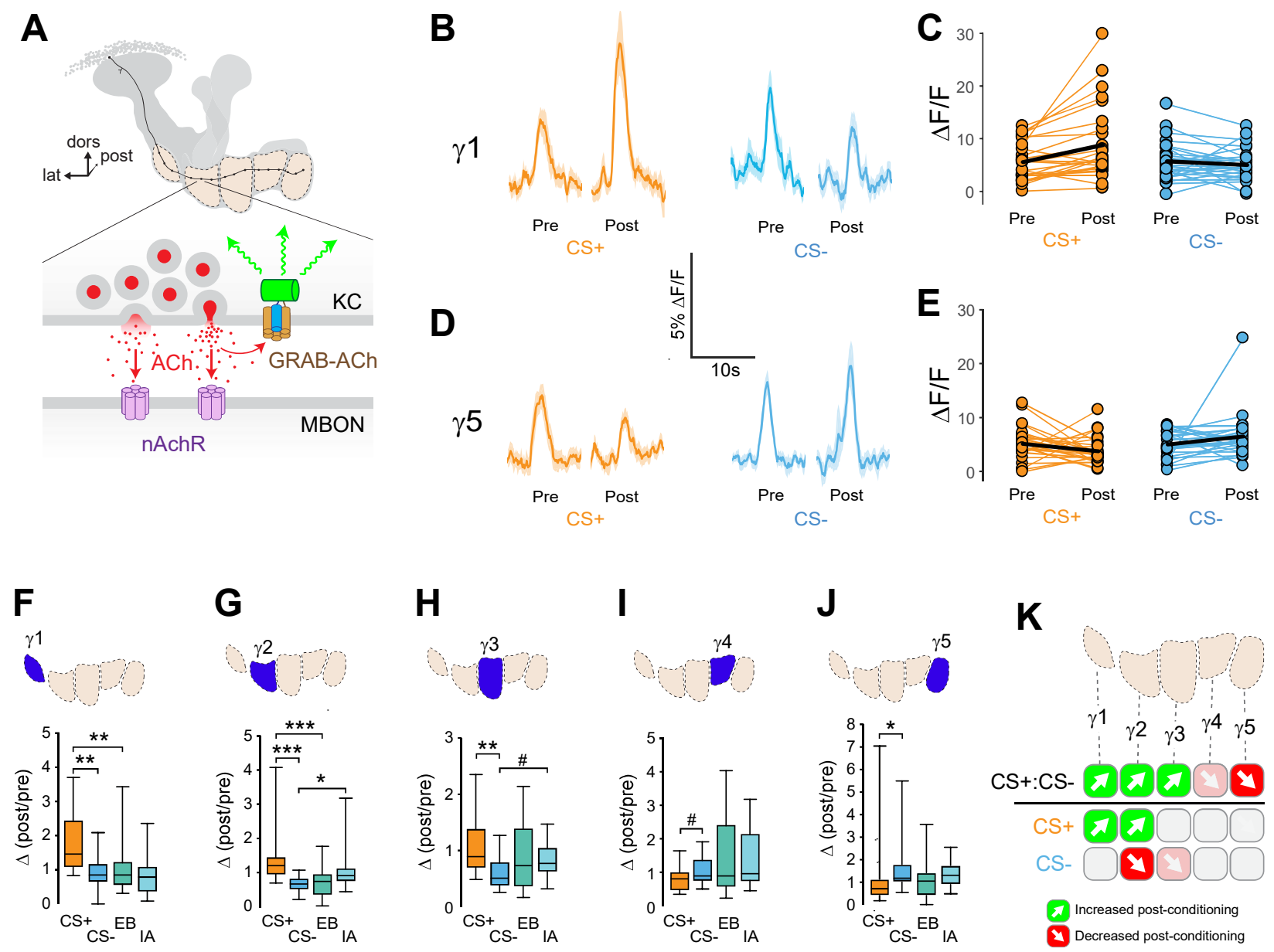

Figure 1. Compartment-specific alterations of $A C h$ release in the MB following appetitive conditioning. (A) Diagram of the GRAB-ACh reporter expressed in presynaptic terminals of MB neurons (Kenyon cells: MB). nAChR: nicotinic acetylcholine receptor; dors: dorsal; lat: lateral, post: posterior; MBON: mushroom body output neuron. (B) Time series traces showing odor-evoked GRAB-ACh responses pre- and post-conditioning. Responses were imaged to both the CS+ (ethyl butyrate: EB) and CS(isoamyl acetate: IA) odor in the $\gamma 1$ compartment, and the line and shading represent the mean \pm SEM. (C) Quantification of the pre- and post-conditioning responses to the CS+ (EB) and CS- (IA) from the $\gamma 1$ compartment from individual animals $(n=27)$, with the mean graphed as a black line. (D) Time series traces imaged from the $\gamma 5$ compartment, graphed as in panel B. (E) Quantification of peak responses from the $\gamma 5$ compartment, graphed as in panel C. (F-J) Change in odor-evoked responses (Post/pre responses), following conditioning (CS+ and CS-) or odor-only presentation (EB and IA). ${ }^{*} \mathrm{p}<0.01$, ${ }^{* *} p<0.001,{ }^{* * *} \boldsymbol{p}<0.0001 ; \boldsymbol{n}=27$ (Kruskal-Wallis/Bonferonni). (F) $\gamma 1$ compartment. (G) $\gamma 2$ compartment. (H) $\gamma 3$ compartment. $\# p=0.0169$. (I) $\gamma 4$ compartment. $\# p=0.0868$. (J) $\gamma 5$ compartment. (K) Summary of plasticity in ACh release across $\gamma$ lobe compartments. Green up arrows indicate increases in the CS+:CS- (1st row) or potentiation of the CS+ response (relative to odor-only controls; 2 nd row), while red down arrows indicate decreases in the CS+:CS- (1st row) or depression of the CS- (relative to odor-only controls; 3rd row). 
pairing of odor with stimulation of PPL1 neurons that are critical for aversive learning (Hige et al., 2015a), suggesting that depression may be a primary mechanism for learning at these synapses (Barnstedt et al., 2016; Cohn et al., 2015; Handler et al., 2019; Owald et al., 2015; Perisse et al., 2016; Sejourne et al., 2011). One synapse downstream, some MBONs exhibit bidirectional responses to conditioning, though the major described mechanism involves a sign change that occurs postsynaptic to the MBs (polysynaptic feedforward inhibition) (Owald et al., 2015; Perisse et al., 2016). To test for the presence, directionality, and variation of presynaptic plasticity across MB axonal compartments, we expressed a synaptic ACh sensor to monitor neurotransmitter release from MB neurons in vivo (Zhang et al., 2019). The geneticallyencoded ACh reporter, GPCR-Activation-Based-ACh sensor (GRAB-ACh) (Fig. 1A) (Jing et al., 2019; Jing et al., 2018; Zhang et al., 2019), was expressed in MB neurons using the 238Y-Gal4 compartments evoked by the CS+ and CS- before and after pairing odor with sucrose (Fig. S1). Responses were compared to those in odor-only control cohorts to determine whether any learning-induced changes resulted from potentiation or depression. We quantified several parameters (Fig. S1), including how the responses changed after conditioning (the within-

111 treatment post/pre). In addition, we compared the CS+ and CS- responses after conditioning

112 (CS:CS-), which mimics the putative comparison the animal makes during associative memory

113 retrieval. Finally, we compared the change in CS+ and CS-, $\Delta$ (post/pre), to their respective 114 odor-only controls to quantify whether they were potentiated or depressed by conditioning, accounting for any sensory adaptation (Figs. 1 F-J, S2).

Appetitive conditioning produced plasticity in ACh release that varied across the axonal 
increased CS+ responses relative to the CS- responses ( $\uparrow \mathrm{CS}+: \mathrm{CS}-)$ in the three most proximal $\gamma$ lobe compartments: $\gamma 1, \gamma 2$, and $\gamma 3$ (Figs. $1 \mathrm{~B}, \mathrm{C}, \mathrm{F}-\mathrm{H}$; S2). In each of these compartments, this

122 potentiated; i.e., following appetitive conditioning, the $\Delta$ (post/pre) CS+ response was larger than

123 the ethyl butyrate (EB) odor-only control, while the CS- did not significantly differ from the

124 isoamyl acetate (IA) odor-only control (Figs. 1F, S2). In the $\gamma 2$ compartment, both the CS+ was

125 potentiated and the CS- depressed relative to the odor-only controls (Figs. 1G, S2). Finally, in

126 the $\gamma 3$ compartment, neither was significantly altered relative to odor-only controls at the

127 Bonferroni-corrected $\alpha=0.01$ level, but there was a strong trend toward depression with the CS-

128 group (Figs. 1H, S2). Thus, there was a spatial gradient of CS+ potentiation in $\gamma 1$, shifting from

129 CS+ potentiation in $\gamma 1$ toward CS- depression in $\gamma 3$, with the spatially-intermediate $\gamma 2$ exhibiting

130 both. This gradient of CS+:CS- plasticity suggests that both the CS+ and CS- contribute to

131 learning by modulating MB output.

In the distal $\gamma 4-\gamma 5$ compartments, appetitive conditioning produced plasticity in the opposite

134 direction to that in the proximal $\gamma$ compartments. In these compartments, the CS+ response was reduced relative to the CS- ( $\downarrow$ CS+:CS-) (Figs. 1D, E, I-J, S2). This effect was significant in

137 compartments, the effect could not be unambiguously assigned to CS+ depression, though

138 there was no evidence of CS- potentiation (Figs. 1 I,J, S2). Overall, appetitive conditioning

139 produced net enhancement of CS+ responsivity in $\gamma 1-\gamma 3$ compartments, which was derived from

140 a proximal-to-distal gradient of CS+ potentiation to CS- depression, and net reduction of CS+

141 responsivity in $\gamma 4-\gamma 5$ (Fig. $1 \mathrm{~K})$. Thus, the plasticity was bidirectional between the proximal and

142 distal axonal compartments. This likely contributes to approach behavior by simultaneously 
143 enhancing the conditioned odor-evoked activation of downstream "approach" circuits and

144 inhibition of "avoidance" MBON circuits.

The above data suggested that appetitive conditioning produced synaptic potentiation in the

149 proximal $\gamma$ lobe compartments. Yet synaptic depression is the main described plasticity

150 mechanism at the MB-MBON synapses following olfactory conditioning (Barnstedt et al., 2016;

151 Modi et al., 2020; Owald et al., 2015; Perisse et al., 2016; Sejourne et al., 2011; Zhang and

152 Roman, 2013; Zhang et al., 2019). In the $\gamma 1$ compartment, where it has been examined in

153 detail with electrophysiology, aversive reinforcement substitution produces synaptic depression

154 (Hige et al., 2015a). Since many of these studies involved aversive conditioning, we reasoned

155 that appetitive and aversive conditioning may produce bidirectional plasticity, with the

156 sign/directionality matching postsynaptic MBON valence. To test this, we examined whether

157 aversive conditioning produced the opposite effect in the same compartments as appetitive

158 conditioning had. ACh release from MB neurons was imaged with GRAB-ACh and flies were

159 trained with an aversive odor-shock conditioning protocol (Fig. 2A). In these experiments, we

160 focused on the $\gamma 2-\gamma 5$ compartments, as the fly was mounted at a higher angle, making the

161 GRAB-ACh signal difficult to simultaneously visualize from $\gamma 1$ along with that of the other

162 compartments. Following aversive conditioning, there was a reduction in the CS+ response

163 relative to the CS- ( $\downarrow$ CS+:CS-) in the $\gamma 2$ and $\gamma 3$ compartments (Fig. $2 \mathrm{C}-\mathrm{H}$ ). This was due to

164 depression in the CS+ response, as the post-conditioning CS+ response was significantly

165 smaller than odor-only controls. The $\gamma 4$ and $\gamma 5$ compartments exhibited no significant change in

166 ACh release (Fig 2 I-J). When compared to appetitive conditioning, aversive stimuli produced 


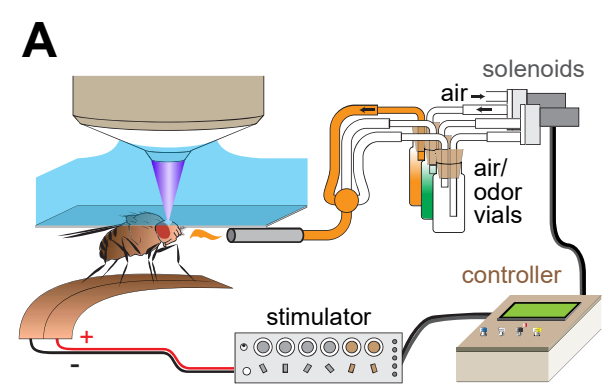

B

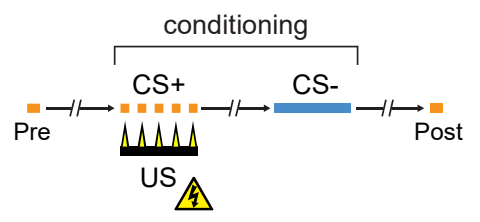

G
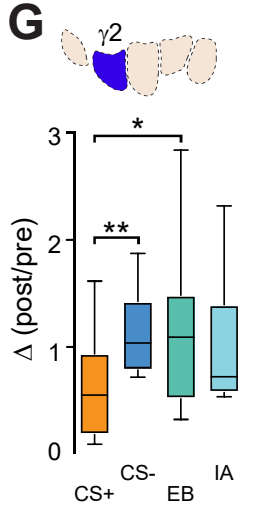
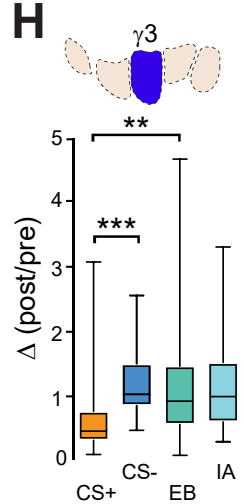

C

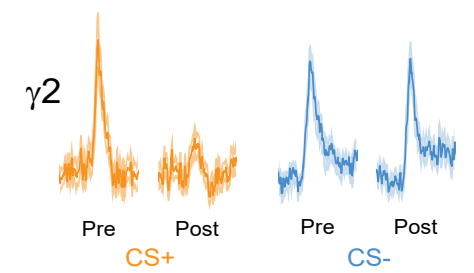

E

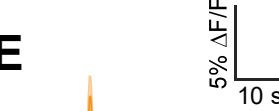

I
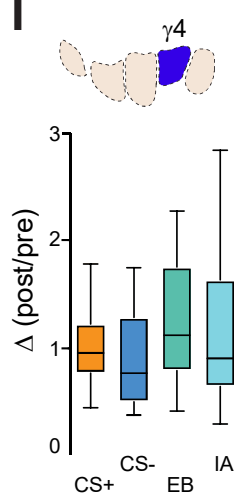

D

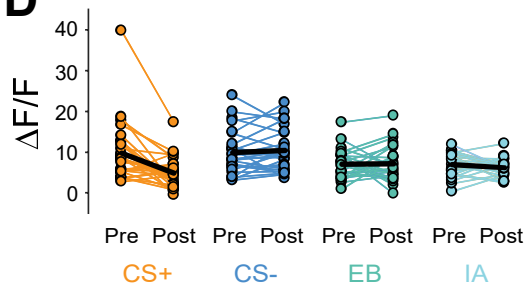

$\mathbf{F}$

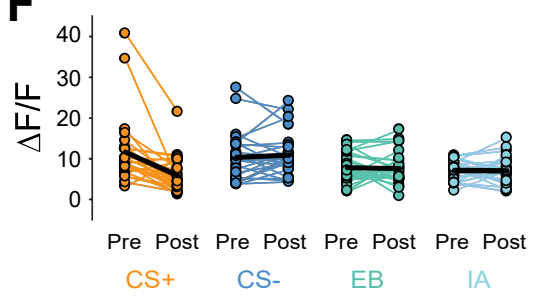

Figure 2. Compartment-specific alterations of $A C h$ release in the $M B$ following aversive conditioning. (A) Diagram of the aversive conditioning apparatus. (B) Aversive conditioning experimental protocol, pairing an odor (the CS+) with an electric shock unconditioned stimulus (US) (6 shocks, 60V). A second odor, the CS- was presented $5 \mathrm{~min}$ after pairing the CS+ and US. One odor was imaged before (Pre) and after (Post) conditioning per animal (CS+ diagrammed here). (C) Time series traces showing odor-evoked GRAB-ACh responses pre- and post-conditioning. Responses were imaged to both the CS+ (ethyl butyrate: EB) and CS- (isoamyl acetate: IA) odor in the $\gamma 2$ compartment, and the line and shading represent the mean \pm SEM. (D) Quantification of the peak pre- and post-conditioning responses to the CS+ $(E B)$ and CS- (IA) from the $\gamma 2$ compartment from individual animals $(n=27)$, with the mean graphed as a black line. (E) Time series traces imaged from the $\gamma 3$ compartment, graphed as in panel B. (F) Quantification of peak responses from the $\gamma 3$ compartment, graphed as in panel C. (G-J) Change in odor-evoked responses (Post/pre responses), following conditioning (CS+ and CS-) or odor-only presentation (EB and IA). ${ }^{*} \mathrm{p}<0.01,{ }^{* *} \mathrm{p}<0.001,{ }^{* * *} \mathrm{p}<0.0001 ; \mathrm{n}=27$

(Kruskal-Wallis/Bonferonni). (G) $\gamma 2$ compartment. (H) $\gamma 3$ compartment. (I) $\gamma 4$ compartment. (J) $\gamma 5$ compartment. (K) Summary of plasticity in ACh release across $\gamma$ lobe compartments. Red down arrows indicate decreases in the CS+:CS- (1st row) or depression of the CS+ (relative to odor-only controls; $2^{\text {nd }}$ row). 
167 plasticity that created a sign flip in the $\gamma 2$ and $\gamma 3$ compartments (Figs. 1K, 2K). Thus, appetitive

168 and aversive conditioning produced bidirectional plasticity across multiple compartments, which 169 was due to localized plasticity within MB $\gamma$ lobe. The aversive conditioning-induced depression

170 likely represents a presynaptic contribution to learning-induced changes in odor responsivity

171 among postsynaptic MBONs (Berry et al., 2018; Hige et al., 2015a; Owald et al., 2015; Zhang et

172 al., 2019).

\section{Presynaptic potentiation relies on the cacophony $\mathrm{Cav} 2.1 \mathrm{Ca}^{2+}$ channel}

Associative learning alters $\mathrm{Ca}^{2+}$ transients in $\mathrm{MB} \gamma$ neurons (Louis et al., 2018), which could

176 influence neurotransmitter release. Major sources of stimulus-evoked intracellular $\mathrm{Ca}^{2+}$ include

177 influx through voltage-sensitive Cav2 channels, which are involved in presynaptic short-term

178 and homeostatic plasticity (Frank et al., 2006; Inchauspe et al., 2004; Ishikawa et al., 2005;

179 Muller and Davis, 2012). To probe the mechanisms of $\mathrm{Ca}^{2+}$-dependent molecular mechanisms

180 underlying presynaptic plasticity, we first knocked down the $\alpha$ subunit of the $\mathrm{Cav}_{2} \mathrm{Ca}^{2+}$ channel

181 encoded by cacophony (Cac), in the mushroom body. Cac was knocked down conditionally in

182 adult MBs with RNAi using the R13F02-Gal4 driver, combined with the ubiquitous temperature-

183 sensitive tub-Gal80 ${ }^{\text {ts }}$ repressor (McGuire et al., 2003) to circumvent any potential for

184 developmental effects (Fig. 3A). RNAi expression was induced four days prior to the

185 experiment, and ACh release from MB neurons was imaged with GRAB-ACh (Jing et al., 2018;

186 Zhang et al., 2019). Control flies (containing R13F02-Gal4, UAS-GRAB-ACh, and tub-Gal80 ${ }^{\text {ts }}$,

187 but lacking a UAS-RNAi) exhibited plasticity across the $\gamma$ lobe in the same spatial patterns as

188 previously observed: there was an increase in relative CS+ responses in the $\gamma 1-\gamma 3$

189 compartments, and a trend toward a CS+ decrease in $\gamma 5$ (Fig. 3 C,E,F, S4). When Cac was

190 knocked down conditionally, odor-evoked ACh release was still observed, demonstrating that 
bioRxiv preprint doi: https://doi.org/10.1101/2021.06.08.447536; this version posted June 10, 2021. The copyright holder for this preprint (which was not certified by peer review) is the author/funder, who has granted bioRxiv a license to display the preprint in perpetuity. It is made available under aCC-BY 4.0 International license.

A
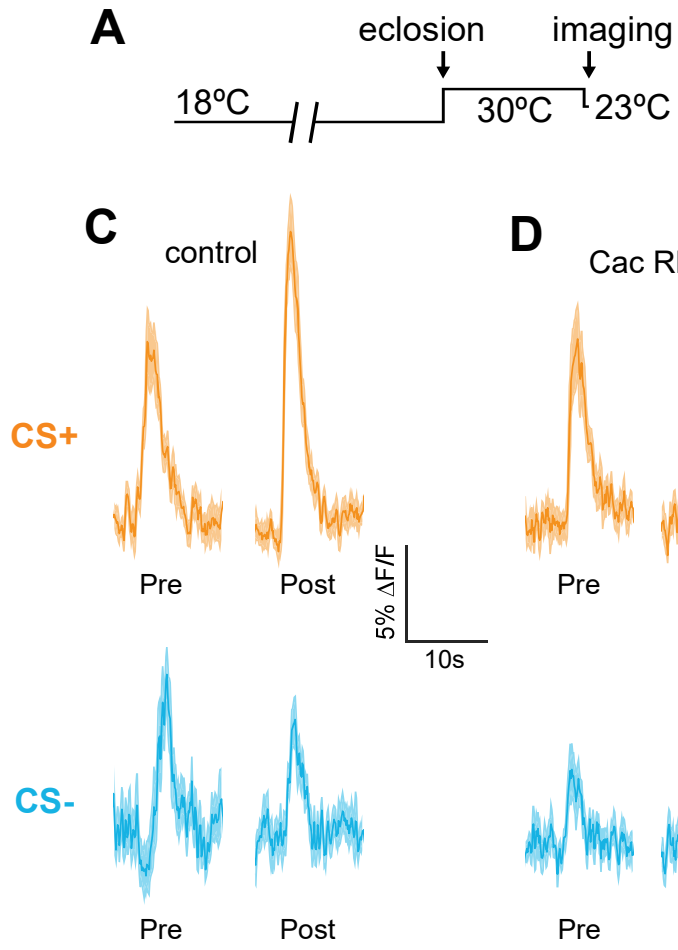
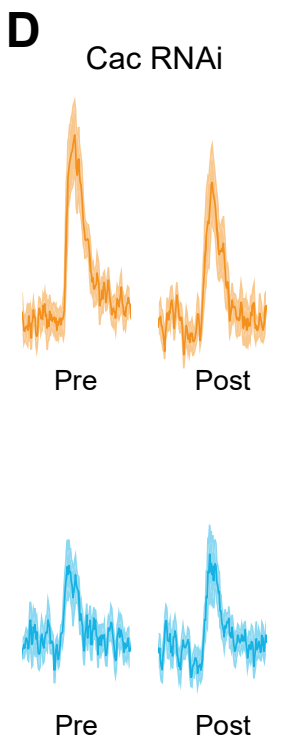

B
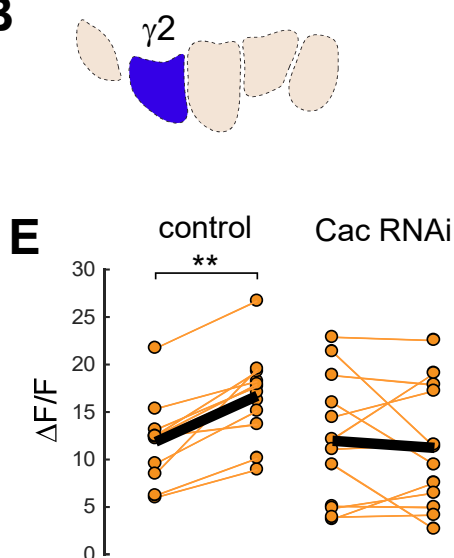

Pre Post Pre Post

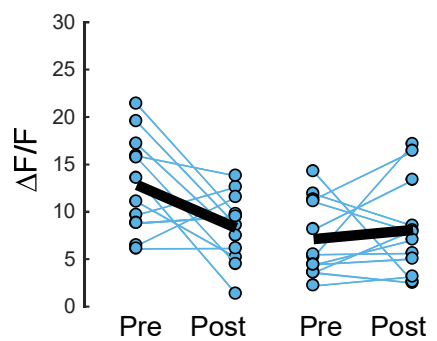

$\mathbf{F}$ Control

$\gamma 1$
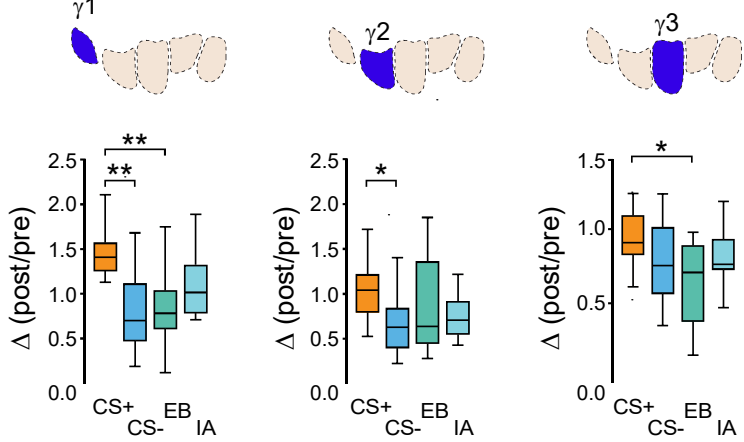
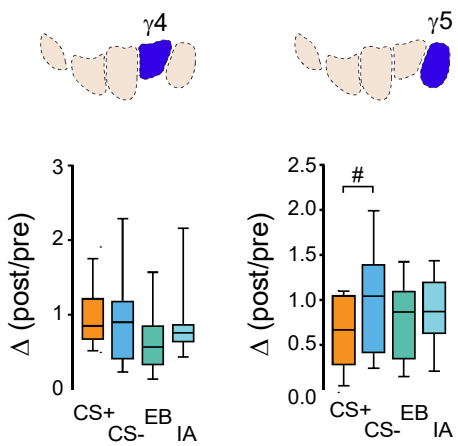

G
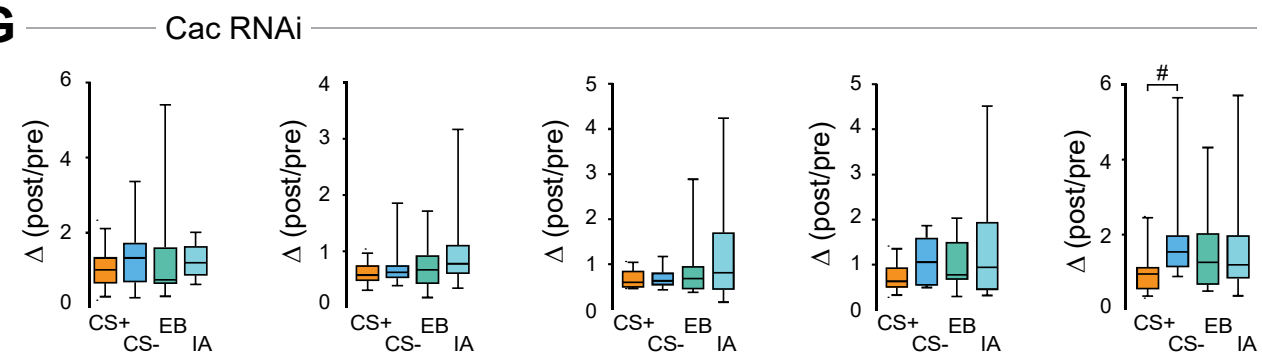

Figure 3. Conditional knockdown of the $\mathrm{Ca}_{\mathrm{v}} 2$ channel $\mathrm{Cac}$ impairs potentiation of $\mathrm{ACh}$ release from the $\mathrm{MB}$ following appetitive conditioning. (A) Diagram of the temperature shifts employed for conditional knockdown of Cac with tub-Gal80ts. (B) Diagram of the MB compartments, highlighting the $\gamma 2$ compartment that was imaged for the data shown in panels C-E. (C) Pre- and post-conditioning CS+ (orange; top) and CS- (blue; bottom) odor-evoked ACh release from the $\gamma 1$ compartment before and after appetitive conditioning, imaged in control animals (w;UAS-GRAB-ACh/+; R13F02-Gal4/UAS-tub-Gal80ts). Time series trace with line and shading representing mean \pm S.E.M. (D) CS+ and CS- odor-evoked ACh release from the $\gamma 1$ compartment in animals with conditional knockdown of Cac (w;UAS-GRAB-ACh/UAS-Cac-RNAi;R13F02-Gal4/UAS-tub-Gal80ts). (E) Pre- and post-conditioning $\Delta$ F/F $\mathrm{CS}+$ and CS- responses in control and Cac knockdown animals. (F) Change in ACh release (post/pre response) following appetitive conditioning (CS+ and CS-) and odor-only presentation (EB: ethyl butyrate; IA: isoamyl acetate) in control animals across the five MB $\gamma$ lobe compartments: $\gamma 1-\gamma 5$ (left to right). (G) Change in ACh release across the five MB compartments in animals with conditional knockdown of Cac. ${ }^{* *} p<0.01,{ }^{*} p<0.01 ; \# p<0.07 ; n=12$. 
synaptic exocytosis remained intact. Yet the CS+ potentiation was lost across the $\gamma 1-\gamma 3$ compartments (Fig. 3 D,G, S4). This demonstrates that potentiation of ACh release to the trained odor - induced by learning - is dependent on the presynaptic Cav2.1 channel Cac.

Data from the appetitive conditioning experiments suggested that potentiation of the CS+ response was dependent on Cac. Interestingly, the trend toward CS+ depression in the most distal $\gamma 5$ compartment remained intact when Cac was knocked down (Fig. 3). This suggests that presynaptic potentiation, but not depression, requires the voltage-sensitive $\mathrm{Ca}_{\mathrm{v}} 2 \mathrm{Ca}^{2+}$ channel cacophony across the MB compartments. To further examine whether depression of the CS+ was affected, we turned to aversive conditioning, which generates robust CS+ depression in the proximal $\gamma$ compartments (Fig. 2). Control flies for conditional knockdown experiments exhibited similar CS+ depression in the proximal $\gamma 2,3$ lobes. Knock down of Cac did not appreciably impair depression of CS+ responses. There was a significant depression in

$204 \gamma 2$, both in terms of CS+:CS- and CS+ relative to odor-only controls (Figs. 4, S5). In $\gamma 1$, there

205 was a trend toward a decrease in the CS+:CS- ratio that matched the controls (Fig. S5). In $\gamma 3$,

206 the difference between the CS+ and CS- (or odor-only control) did not reach significance, but

207 there was a trend in the same direction as the controls (Fig. S5). Overall, these data

208 demonstrate that Cac is not required for learning-induced depression of ACh release.

210 Post-conditioning odor contrast and maintenance of odor responses are dependent on $211 \quad \mathbf{I P}_{3}$ signaling

$212 \mathrm{Ca}^{2+}$ release from the endoplasmic reticulum (ER) is a major source of stimulus-evoked $\mathrm{Ca}^{2+}$ in 213 neurons, including MB neurons, and modulates various forms of synaptic/homeostatic plasticity 
214 (Handler et al., 2019; James et al., 2019; Taufiq et al., 2005). Therefore, we reasoned that

215 inositol triphosphate receptor $\left(\mathrm{IP}_{3} \mathrm{R}\right)$ mediated $\mathrm{Ca}^{2+}$ release may contribute to presynaptic

216 plasticity across MB compartments. To test this, we conditionally knocked down the $I P_{3} R$ in the

217 adult MB with RNAi. GRAB-ACh was expressed in the MB (as above) while conditionally

218 knocking down $I P_{3} R$ (Fig. 4, S5). For these experiments, flies were aversively conditioned (IP ${ }_{3} R$

219 knockdown impairs feeding under the microscope, precluding appetitive conditioning).

220 Knockdown of $\mathrm{IP}_{3} \mathrm{R}$ eliminated the post-conditioning contrast between the CS+ and CS- (i.e.,

221 the difference between the CS+ and CS-) (Fig. 4, S5). This was due to increased adaptation to

222 the odors (reduction in post-conditioning odor responses). This occurred in the CS- and both

223 odor-only control groups, bringing them down to a similar level to the level of the CS+ group

224 (Fig. 4 C-E). Thus, in normal conditions, release of $\mathrm{Ca}^{2+}$ from the $E R$ via IP ${ }_{3} \mathrm{R}$ is necessary to

225 maintain odor responsivity upon repeated odor presentations. Loss of $I P_{3} R$ renders the MB

226 neurons more susceptible to adaptation, reducing the contrast between the CS+ - which

227 exhibits depression following aversive learning - and the other odor(s).

\section{Compartmentalized plasticity propagates into downstream mushroom body output}

neurons

231 Since ACh release from each compartment provides input to unique postsynaptic mushroom

232 body output neurons, the presynaptic plasticity observed in each compartment should be

233 mirrored in the respective postsynaptic MBON(s) innervating that compartment. To test this, we

234 imaged $\mathrm{Ca}^{2+}$ responses in MBONs with GCaMP and examined the effect of appetitive

235 conditioning. Four sets MBONs were tested, each innervating and receiving cholinergic input

236 from a distinct MB $\gamma$ lobe compartment: $\gamma 1$ pedc $>\alpha / \beta, \gamma 2 \alpha^{\prime} 1, \gamma 3 / \gamma 3 \beta^{\prime} 1$, and $\gamma 5 \beta^{\prime 2} 2 a$ (Fig. 5A).

237 Within the $\gamma$ lobe, these neurons innervate the $\gamma 1, \gamma 2, \gamma 3$, and $\gamma 5$ compartments, respectively 

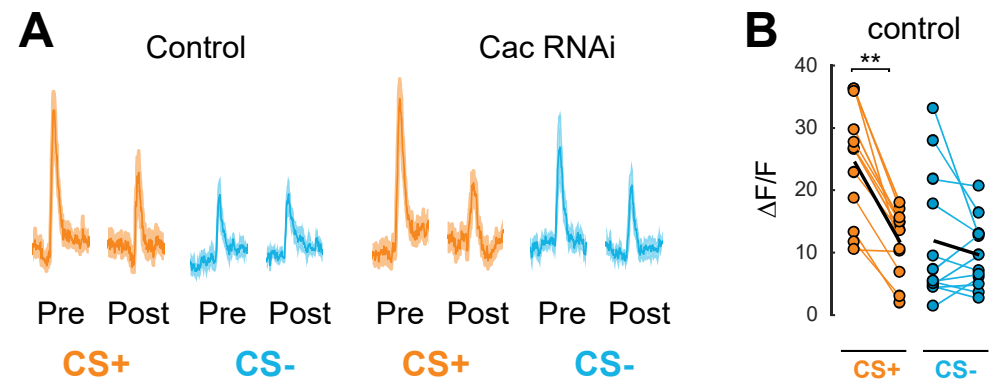

Cac RNAi

C

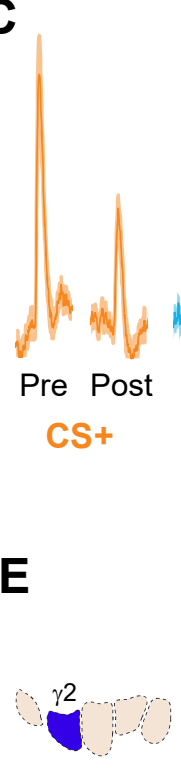

IP ${ }_{3}$ RNAi

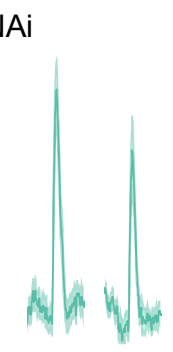

Pre Post

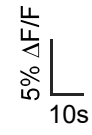

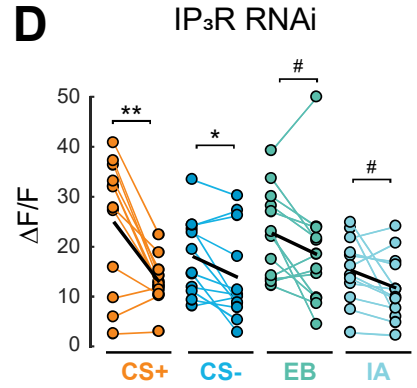

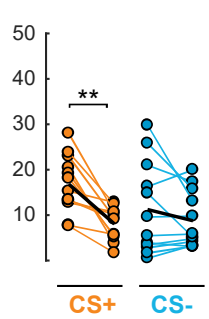

CS-

EB
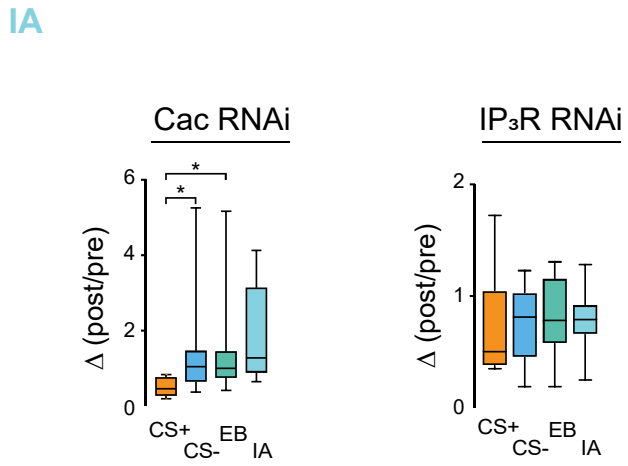

Figure 4. Cac and $I P_{3} R$ exert distinct effects on synaptic plasticity and maintenance of olfactory responses following aversive conditioning. (A) Pre- and post-conditioning CS+ and CS- odor-evoked ACh release in control and Cac RNAi flies. Time series trace with line and shading representing mean \pm S.E.M. (B) Pre- and post-conditioning $\triangle F / F$ CS+ and CS- responses in control and Cac knockdown animals. Each thin line connects the pre- (left) and post-conditioning response (right) for one animal. The thick black line represents the mean. (C) ACh release in $I P_{3} R$ knockdown animals for trained odors (CS+ and CS-) as well as the respective odor-only controls (ethyl butyrate [EB] and isoamyl acetate $[I A])$. (D) $\Delta F / F$ responses in $I_{3} R$ knockdown flies. (E) Change in ACh release (post/pre response) following aversive conditioning (CS+ and CS-) and odor-only presentation (EB and IA) in controls, as well as flies with conditional Cac and $\mathrm{IP}_{3} \mathrm{R}$ knockdown. 
238 (Fig. 5 B-F). The $\gamma 1$ pedc $>\alpha / \beta$ MBON exhibited a significant elevation of the CS+ response

239 relative to the CS- ( $\uparrow \mathrm{CS}+\mathrm{CS}-)(\mathrm{Fig} .5 \mathrm{H})$. This was due to a potentiation of the CS+ response,

240 as the post-conditioning CS+ response was significantly larger than the corresponding odor-only

241 control. The $\gamma 2 \alpha^{\prime} 1 \mathrm{MBON}$ also exhibited an increase in the CS+:CS- ratio following conditioning

242 (Fig. 5I). In this neuron, the plasticity could not be unambiguously attributed to purely CS+

243 potentiation or CS- depression. The $\gamma 3 / \gamma 3 \beta^{\prime} 1$ MBONs exhibited an increase in the CS+:CS- that

244 was due to potentiation of the CS+ response (Fig. 5J). Note that these neurons are not parsed

245 with available drivers and were imaged as a pair. Presynaptically, the $\gamma 3$ compartment exhibited

246 a depression in the CS- response, suggesting that the potentiation in the MBON CS+ response

247 may emanate either from the $\beta$ ' 1 inputs or modulation via polysynaptic circuit interactions.

248 Finally, appetitive conditioning produced plasticity in the opposite direction in the $\gamma 5 \beta^{\prime 2} 2 a \mathrm{MBON}$;

249 this neuron exhibited a decrease in the CS+ response relative to the CS- ( $\downarrow$ CS+:CS-) (Fig. 5K).

250 In each case, the directionality of the plasticity (CS+:CS-) matched that observed in ACh

251 responses in the presynaptic compartment. Thus, compartmentalized, presynaptic plasticity in

252 neurotransmitter release from the MB compartments likely plays a role in modulating the MBON

253 responses following learning.

255 Isolation of timing effects reveals CS- specific depression in the $\gamma 3$ compartment

256 Synaptic depression in ACh release in the $\gamma 2$ and $\gamma 3$ compartments following appetitive

257 conditioning was unique in that, in wild-type animals, it involved plasticity to the CS- (Figs. 2

$258 \mathrm{G}, \mathrm{H}, \mathrm{S} 3$ ). This raised the question of whether the simple act of presenting an odor 30 seconds

259 after the offset of US pairing - the time at which the CS- is presented in the conditioning

260 paradigm - is sufficient to alter ACh release. To test this, we compared the results from the

261 discriminative CS+/CS- imaging assay (Figs. 2, S3) with a single-odor paradigm (Fig. 6A). Flies 
A

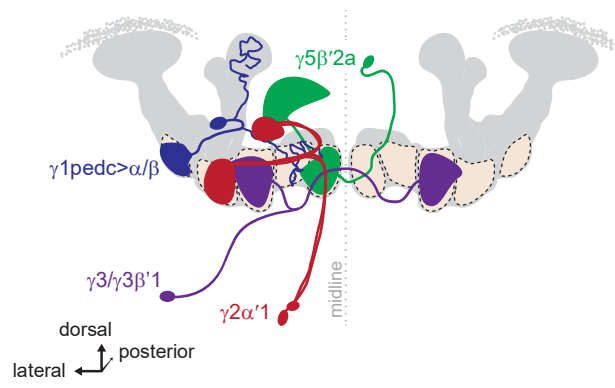

B

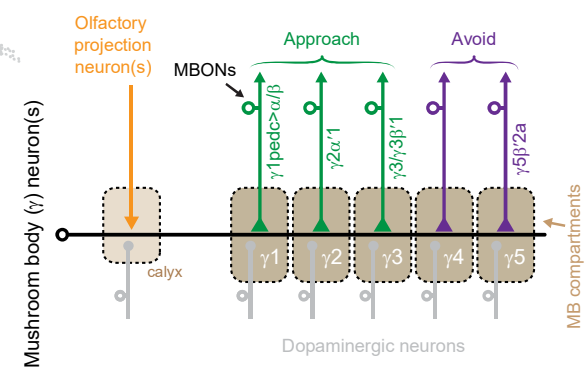

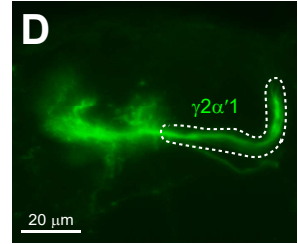

H

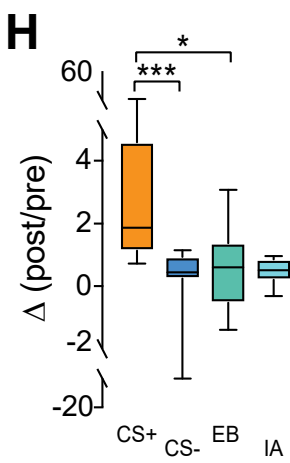

I

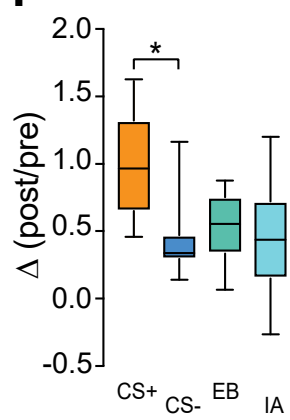

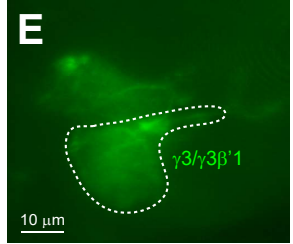

$\mathbf{J}$

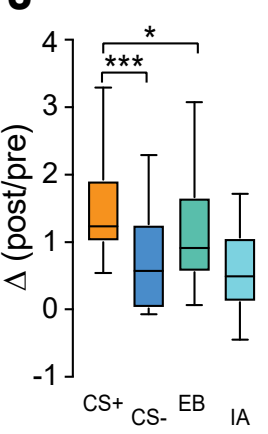

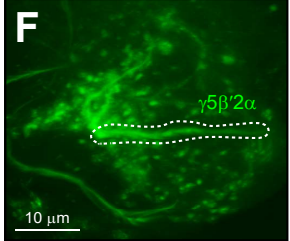

K

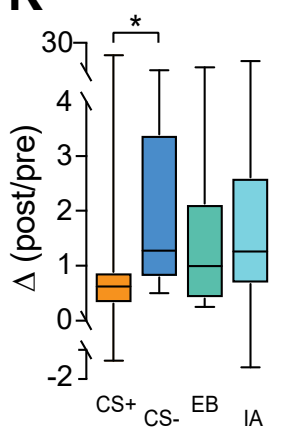

Figure 5. Plasticity in $\mathrm{MBON} \mathrm{Ca}{ }^{2+}$ responses mirrors compartmental plasticity in the $\mathrm{MB}$ neurons. (A) Diagram of MBONs innervating specific $\gamma$ lobe compartments, viewed from a frontal plane. Each MBON is bilaterally paired, though only one is drawn here for visual clarity. (B) Circuit diagram of the dopaminergic neurons and MBONs in each compartment, as well as the putative valence associated with each compartment/MBON. (C-F) Diagrams of the $\gamma 1$ pedc $>\alpha / \beta, \gamma 2 \alpha^{\prime} 1, \gamma 3$, and $\gamma 5 \beta^{\prime} 2 a$ MBONs, respectively, drawn unilaterally in isolation. (G-J) Representative confocal images of the $\gamma 1$ pedc $>\alpha / \beta$, $\gamma 2 \alpha^{\prime} 1, \gamma 3$, and $\gamma 5 \beta^{\prime} 2 a$ MBONs, respectively. The region of interest circumscribed for quantification (neuropil or an efferent neurite) is drawn with a dotted white line. lat: lateral, dors: dorsal, post: posterior. (K-N) Change in odor-evoked responses (Post/pre responses), following conditioning (CS+ and CS-). ${ }^{* * *} p<0.001,{ }^{*} p<0.01 ; n=12$ (Kruskal-Wallis/Bonferroni). 
expressing GRAB-ACh in the MB via the $238 \mathrm{Y}-\mathrm{Gal} 4$ driver were presented with an odor and sucrose, in a similar manner to the standard discriminative appetitive conditioning protocol, except that the CS+, CS-, or US was omitted (Fig. 6A, S6). We compared the change in responses to that odor across the three protocols in all five compartments (Fig. 6C, S3). This revealed several major facets of plasticity in ACh release following appetitive conditioning. First,

267 discriminative training is necessary for the potentiation in $\gamma 1$ and $\gamma 2$, which was lost in singleodor CS/US training (protocol \#1) (Fig. 6A,C, S6). In addition, when omitting the CS+, only the $\gamma 3$ compartment revealed significant timing effects (Fig. 6 B,C, S6); presenting sucrose prior to

270 presentation of an odor in the normal CS- time slot (protocol \#2) resulted in a significantly

271 smaller response than CS/US pairing, as well as a trend toward depression relative to the odor-

272 only group. Therefore, the backward temporal contingency of the odor and sucrose

273 presentation likely underlies the depression of odor-evoked responses in the $\gamma 3$ region observed 274 with discriminative CS+/CS- learning (Fig. 2H). Overall, these data demonstrate that the $\gamma 3$

275 compartment is particularly important for the temporal comparison of the CS+ and CS-, which is 276 critical for discriminative learning (a possibility we explore further below).

279 The unique role of the $\gamma 2$ and $\gamma 3$ compartments in encoding CS- plasticity led us to question the

280 behavioral roles of the MBONs that receive input from these compartments (Figs. 1, 6A-C).

281 With the exception of the $\gamma 1$ pedc $>\alpha / \beta$ (Perisse et al., 2016), the involvement of these MBONs in

282 appetitive memory is unclear. To test whether the MBONs innervating the $\gamma 2$ and $\gamma 3$

283 compartments mediate appetitive memory, we carried out behavioral appetitive classical

284 conditioning, blocking synaptic transmission from MBONs with Shibire ${ }^{\text {ts }}\left(\right.$ Sht $\left.^{\text {ts}}\right)$ (McGuire et al.,

285 2001) (Fig. 6 D,E). Blocking the $\gamma 2 \alpha^{\prime} 1 \mathrm{MBON}$ did not significantly impair performance in 


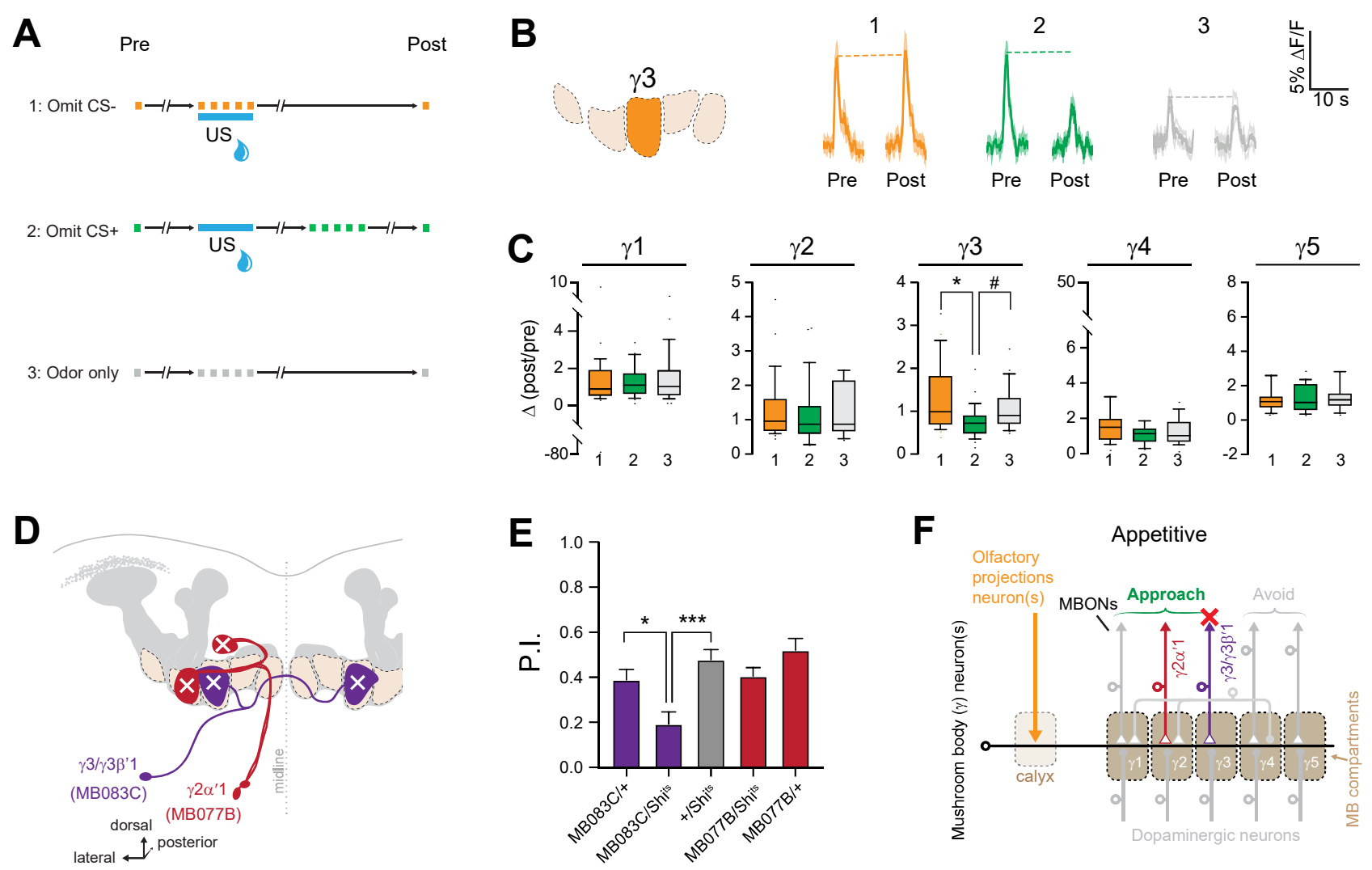

Figure 6. MB $\gamma 3$ plasticity encodes appetitive timing (CS-) effects, and output neurons from this region are necessary for appetitive learning. (A) Diagram of the training paradigms utilized for ACh imaging experiments. (B) Time series traces showing odor-evoked GRAB-ACh responses from the $\gamma 3$ compartment with all three protocols. (C) Quantification of the odor-evoked post/pre responses from each $\gamma$ lobe compartment. ${ }^{*} \mathrm{p}<0.01$, $\# \mathrm{p}=0.016 ; n=27$ (Kruskal-Wallis/Bonferroni). (D) Anatomical Diagram of the $\gamma 2 \alpha^{\prime} 1$ (MB077B-Gal4) and $\gamma 3 / \gamma 3 \beta^{\prime} 1$ (MB083C-Gal4) MBONs. (E) Behavioral appetitive conditioning in flies, silencing either $\gamma 2 \alpha^{\prime} 1$ or $\gamma 3 / \gamma 3 \beta^{\prime} 1$ MBONs with Shibirets (Shits), compared to heterozygous Gal4/+ and UAS/+ controls. P.I.: Performance Index. ${ }^{*} p<0.05,{ }^{* * *} p<0.0005$ (ANOVA/Sidak); $n=16$. (F) Circuit diagram of the output of the $\gamma 2 \alpha^{\prime} 1$ and $\gamma 3 / \gamma 3 \beta^{\prime} 1 \mathrm{MBONs}$. 
appetitive conditioning. Therefore, while activation of the $\gamma 2 \alpha^{\prime} 1 \mathrm{MBON}$ drives approach

287 behavior (Aso et al., 2014b) and the neuron is necessary for aversive memory (Berry et al.,

288 2018), it is not crucial for appetitive learning in the otherwise intact nervous system. In contrast,

289 blocking synaptic transmission from the $\gamma 3 / \gamma 3 \beta^{\prime} 1$ MBONs significantly impaired appetitive

290 conditioning performance (Fig. 6E). This demonstrates that the output of the $\gamma 3 / \gamma 3 \beta^{\prime} 1$ MBONs is

291 necessary for normal appetitive short-term memory (Fig. 6F). These neurons convey the output

292 of the MB $\gamma 3$ compartment to the crepine and superior medial protocerebrum (where they

293 innervate interneurons that project to the fan-shaped body and lateral accessory lobe further

294 downstream), as well as provide direct contralateral MB feedback and form polysynaptic

295 feedback loops via MB-innervating PAM dopaminergic neurons and other MBONs (Scaplen et

296 al., 2021; Xu et al., 2020). These multi-layered connections provide several routes through

297 which they could modulate behavioral output following learning. Overall, the present data

298 suggest that the $\gamma 3 / \gamma 3 \beta^{\prime} 1$ MBONs receive input from an MB compartment with unique

299 physiology, and represent a key node through which discriminative effects influence appetitive

300 memory and decision-making.

\section{$\underline{\text { Discussion }}$}

303 Compartmentalized plasticity in neurotransmitter release expands the potential computational

304 capacity of learning circuits. It allows a set of odor-coding mushroom body neurons to bifurcate

305 their output to different downstream approach- and avoidance-driving downstream output

306 neurons, independently modulating the synaptic connections to alter action selection based on

307 the conditioned value of olfactory stimuli. The MB modifies the encoded value of olfactory

308 stimuli through bidirectional plasticity in odor responses, which are compartment-specific along

309 the axons. The CS+ and CS- drive unique patterns of plasticity in each compartment, 
demonstrating that olfactory stimuli are reweighted differently across compartments following

311 learning, depending on the temporal associations of the stimuli. Different molecular

312 mechanisms regulate the potentiation of trained odor responses ( $\mathrm{Cav} 2 / \mathrm{Cac})$ and maintenance

313 of responsivity over time $\left(\mathrm{IP}_{3} \mathrm{R}\right)$. Finally, one set of $\gamma$ output neurons, the $\gamma 3 / \gamma^{3} \beta^{\prime} 1 \mathrm{MBONs}$, is

314 particularly important for appetitive short-term memory.

316 The present data reveal learning-induced, bidirectional plasticity of ACh release in the MB

317 neurons following conditioning with naturalistic stimuli in vivo, which was compartmentally-

318 localized and coherent with the innate valence of the MBON innervating the compartment.

319 Notably, the $\gamma 2$ and $\gamma 3$ compartments, which relay information to approach-promoting MBONs

320 (Aso et al., 2014b), exhibited enhanced CS+:CS- responses to appetitive conditioning, and

321 conversely reduced CS+:CS- following aversive conditioning. This was observed within 5

322 minutes of conditioning, a time point consistent with short-term memory in behavioral assays.

323 Previous studies have described short-term, heterosynaptic depression in the $\gamma 1$ pedc MBON

324 following reinforcement substitution via PPL1 dopaminergic neuron stimulation (Hige et al.,

$3252015 a)$ and changes in odor-evoked $\mathrm{Ca}^{2+}$ responses following olfactory classical conditioning

326 (Perisse et al., 2016). Aversive conditioning has also been shown to decrease neurotransmitter

327 release from the MB neurons (Zhang and Roman, 2013; Zhang et al., 2019). Indirect evidence,

328 via $\mathrm{Ca}^{2+}$ imaging in presynaptic MB neurons, has suggested that increases in presynaptic

329 neurotransmission could be associated with learning. Specifically, reinforcement substitution by

330 pairing odor with stimulation of appetitive PAM dopaminergic neurons potentiates odor-evoked

331 cytosolic $\mathrm{Ca}^{2+}$ transients across the MB (Boto et al., 2014). In addition, appetitive conditioning

332 with naturalistic odor + sucrose pairing increases odor-evoked cytosolic $\mathrm{Ca}^{2+}$ transients in $\mathrm{MB}$

333 neurons (Louis et al., 2018). However, all MB compartments exhibit plasticity with uniform 
334 directionality; short-term aversive conditioning produces no detectable change and appetitive

335 conditioning uniformly elevates odor-evoked responses across the $\gamma 1-\gamma 5$ compartments. Thus,

336 this effect is not selective for subcellular MB compartments that connect to the "aversive" or

337 "appetitive" MBONs. More compartmentalized effects have been observed with other

338 manipulations - presentation of sucrose alters synaptically-localized $\mathrm{Ca}^{2+}$ transients in a

339 compartmentalized manner (Cohn et al., 2015), as does stimulation of $\gamma 4$-innervating

340 dopaminergic circuits (Handler et al., 2019).

342 This study revealed two major mechanisms regulating the spatial patterns of compartmentalized

343 plasticity across the MB compartments: a Cac-dependent CS+ potentiation and an $\mathrm{IP}_{3} \mathrm{R}-$

344 dependent maintenance of sensory responses. This suggests that different sources of

345 intracellular $\mathrm{Ca}^{2+}$ play different roles in regulating $\mathrm{MB}$ synaptic responses. Cac is the pore-

346 forming subunit of the voltage-sensitive, presynaptic Cav2 $\mathrm{Ca}^{2+}$ channel in Drosophila. Cav2

347 channels regulate several forms of synaptic plasticity, including paired-pulse facilitation,

348 homeostatic plasticity, and long-term potentiation (Frank et al., 2006; Inchauspe et al., 2004;

349 Nanou et al., 2016). Our data suggests that these channels regulate the spatial patterns of

350 learning-induced plasticity in the MB unidirectionally, with Cac underlying potentiation but not

351 depression. Cav2 channel activity is modulated by presynaptic calcium and G protein-coupled

352 receptor activity (Zamponi and Currie, 2013), and channel localization in the active zone

353 dynamically regulates synaptic strength (Gratz et al., 2019; Lubbert et al., 2019). These

354 mechanisms may play a role in increasing CS+ responses following appetitive conditioning, as

355 activity in $\mathrm{MB}$ neurons results in increased intracellular $\mathrm{Ca}^{2+}$ and dopaminergic neurons

356 innervating the MB activate receptors that are important for memory formation (Boto et al.,

357 2014; Cohn et al., 2015; Gervasi et al., 2010; Kim et al., 2007; Schwaerzel et al., 2003; Tomchik

358 and Davis, 2009). Baseline stimulus-evoked neurotransmitter release in Cac knockdown was 
maintained, likely mediated by either residual Cac expression or compensation by other intracellular $\mathrm{Ca}^{2+}$ channels/sources. In contrast to potentiation, $\mathrm{IP}_{3} \mathrm{R}$ was necessary to maintain normal odor responsivity when odors were presented on multiple trials (i.e., across pre/post odor presentations). This is consistent with the role of $\mathrm{IP}_{3} \mathrm{R}$ in maintenance of presynaptic

363 homeostatic potentiation at the neuromuscular junction (James et al., 2019). In addition,

364 dopaminergic circuits associated with reward learning drive release of $\mathrm{Ca}^{2+}$ from the

365 endoplasmic reticulum when activated with MB neurons in a backward pairing paradigm ex vivo, 366 potentiating MB $\gamma 4$ connections with the respective $\gamma 4$ MBON (Handler et al., 2019).

Alterations of MBON activity following learning are likely the product of both synaptic plasticity at the MB-MBON synapses and indirect circuit effects, such as feedforward inhibition (Aso et al.,

370 2014a; Cervantes-Sandoval et al., 2017; Perisse et al., 2016). Polysynaptic inhibitory

371 interactions can convert depression from select MB compartments into potentiation in MBONs

372 following learning. In one established example, reduction of odor-evoked responses in the

373 GABAergic $\gamma 1$ pedc MBON following aversive conditioning disinhibits the downstream $\gamma 5 \beta^{\prime} 2 a$

374 MBON (Owald et al., 2015; Perisse et al., 2016). It is unclear whether this mechanism

375 generalizes to other MB compartments. The present data demonstrates that learning drives

376 potentiation and depression of ACh release across multiple MB compartments, providing a

377 direct mechanism for altering MBON responses. Importantly, by comparing the CS+ and CS-

378 responses to those of untrained odors, we ascribed differences between the CS+ and CS- to

379 potentiation or depression in absolute terms within each compartment. This uncovered an

380 additional layer of spatial regulation of plasticity in the $\gamma 1-\gamma 3$ compartments: a gradient of CS+

381 potentiation to CS- depression following appetitive conditioning, which is elaborated in greater 
detail below. In addition, it revealed that the $\mathrm{IP}_{3}$-dependent loss of $\mathrm{CS}+/ \mathrm{CS}$ - contrast was due, at least in large part, to alterations in olfactory adaptation.

The CS+/CS- relationship changed in a linear gradient down the $\gamma 1-\gamma 3$ compartments following appetitive conditioning. Appetitive conditioning increased CS+ responses in the $\gamma 1$ compartment, while decreasing the CS- responses in the $\gamma 3$ compartment. The $\gamma 2$ compartment yielded a mix of these responses. These patterns of plasticity have the net effect of increasing the relative response to the CS+ odor. Since the MBONs postsynaptic to these compartments drive behavioral approach (Aso et al., 2014b), these patterns of plasticity would bias the 391 animal's behavior toward approach of the CS+ if the animal faced both odors simultaneously.

392 Such a situation would occur at the choice point of a T-maze during retrieval in a classical 393 conditioning assay. This further suggests loci where for CS+ and CS- plasticity, which are 394 suggested by behavioral data indicating that temporal/CS- information contribute to behavioral 395 memory (Handler et al., 2019; Konig et al., 2018; Tanimoto et al., 2004; Tully and Quinn, 1985). 396 This is physiologically reflected in plasticity in ACh release to the CS+ and/or CS- across

397 multiple compartments. For instance, the $\gamma 2$ and $\gamma 3$ compartments exhibited a depression in 398 ACh release to the CS-. Therefore, consequences to the specific timing of odor-evoked 399 responses prior to or after the delivery of the US play a key role in memory formation, with 400 bidirectional plasticity forming within the MB neurons based on timing events, valence of the US, 401 and local dopamine signaling (Handler et al., 2019; Konig et al., 2018; Tanimoto et al., 2004; 402 Yamagata et al., 2016). 
404 MBONs innervating the $\gamma$ lobe drive approach/avoidance behavior when stimulated (Aso et al.,

$4052014 b)$. Despite the approach-promoting valence of the $\gamma 2 \alpha^{\prime} 1$ and $\gamma 3 / \gamma 3 \beta^{\prime} 1$ MBONs, only the

$406 \gamma 3 / \gamma 3 \beta^{\prime} 1$ produced a loss-of-function phenotype in appetitive conditioning. This suggests that

407 either the $\gamma 2 \alpha^{\prime} 1 \mathrm{MBONs}$ are uninvolved in appetitive learning (despite exhibiting learning-related

408 plasticity), or that redundancy and/or different weighting across approach-promoting MBONs,

409 renders the system resilient to silencing some of them. Blocking synaptic output of $\gamma 3 / \gamma 3 \beta^{\prime} 1$

410 reduced appetitive conditioning performance, suggesting that these neurons play a particularly

411 important role in appetitive learning.

413 Overall, plasticity between MB neurons and MBONs may guide behavior through biasing

414 network activation to alter action selection in a probabilistic manner. Appetitive conditioning

415 drives compartmentalized, presynaptic plasticity in MB neurons that correlates with postsynaptic

416 changes in MBONs that guide learned behaviors. Prior studies documented only depression at

417 these synapses at short time points following conditioning (Hige et al., 2015a; Zhang and

418 Roman, 2013; Zhang et al., 2019). Here we observed both potentiation and depression in ACh

419 release in the $\mathrm{MB}$, suggesting that bidirectional presynaptic plasticity modulates learned

420 behaviors. These bidirectional changes likely integrate with plasticity at downstream circuit

421 nodes that also undergo learning-induced plasticity to produce network-level alterations in odor

422 responses across the olfactory pathway following salient events. Thus, plasticity in ACh release

423 from MB neurons function to modulate responsivity to olfactory stimuli features across graded

424 plasticity maps down the mushroom body axons. 
427 Fly Strains. Flies were fed and maintained on a standard cornmeal agar food mixture on a

428 12:12 light:dark cycle. The 238Y-Gal4 driver was selected for expression intensity in MB

429 neurons (Louis et al., 2018). MBON drivers were selected from the FlyLight and split-Gal4

430 collections (R12G04, MB077b, and MB083c) (Jenett et al., 2012; Pfeiffer et al., 2010). The

$431 \quad \gamma 5 \beta^{\prime 2} 2 a$ LexA MBON driver was a generated by Krystyna Keleman (Zhao et al., 2018). RNAi

432 lines were obtained from the VDRC (Cac: 101478) (Dietzl et al., 2007) and TRiP collections

$433\left(\mathrm{IP}_{3} \mathrm{R} /\right.$ itpr. 25937) (Perkins et al., 2015) and crossed into flies expressing R13F02-Gal4 and tub-

434 Gal80 ${ }^{\text {ts }}$ (McGuire et al., 2003). Final experimental genotypes were: Cac (w;UAS-GRAB-

435 ACh/UAS-Cac-RNAi;R13F02-Gal4/UAS-tub-Gal80 ts) and IP ${ }_{3} R(w$, UAS-GRAB-ACh/UAS-tub-

436 Gal80"s;:R13F02-Gal4;UAS-IP3R-RNAi), compared to genetic controls (w; UAS-GRAB-

437 ACh/+;R13F02-Gal4/UAS-tub- Gal80's).

438 Fly preparation for in vivo $\mathbf{C a}^{2+}$ imaging. Flies were briefly anesthetized, placed in a

439 polycarbonate imaging chamber, and fixed with myristic acid (Sigma-Aldrich). The proboscis

440 was fixed in the retracted position, except for appetitive conditioning experiments (as noted

441 below). A cuticle window was opened, and the fat and tracheal air sacs were carefully removed

442 to allow optical access to the brain. The top of the chamber was filled with saline solution (103

$443 \mathrm{mM} \mathrm{NaCl}, 3 \mathrm{mM} \mathrm{MBI}, 5 \mathrm{mM}$ HEPES, $1.5 \mathrm{mM} \mathrm{CaCl}_{2}, 4 \mathrm{mM} \mathrm{MgCl} \cdot 6 \mathrm{H}_{2} \mathrm{O}, 26 \mathrm{mM} \mathrm{NaHCO}, 1 \mathrm{mM}$

$444 \mathrm{NaH}_{2} \mathrm{PO}_{4} \cdot \mathrm{H} 2 \mathrm{O}, 10 \mathrm{mM}$ trehalose, $7 \mathrm{mM}$ sucrose, and $10 \mathrm{mM}$ glucose), which was perfused

445 over the dorsal head/brain at $2 \mathrm{~mL} / \mathrm{min}$ via a peristaltic pump.

446 In vivo imaging. GRAB-ACh (Jing et al., 2019; Jing et al., 2018; Zhang et al., 2019) was driven

447 in the MB neurons, using the 238Y driver. Within the MB neurons, ROls were drawn around

448 five $\gamma$ lobe compartments $(\gamma 1-5)$ within a single imaging plane for appetitive, and $(\gamma 2-5)$ for

449 aversive. Imaging was performed with a Leica TCS SP8 confocal microscope utilizing

450 appropriate laser lines and emission filter settings. Odors were delivered with an airstream for

451 1s $(60 \mathrm{~mL} / \mathrm{min}$ flow rate) by directing the air flow with solenoid valves between an empty vial (air) 
452 to another containing $1 \mu \mathrm{L}$ odorant spotted on filter paper. Odor-evoked responses were

453 calculated as the baseline normalized change in fluorescence $(\Delta F / F)$, using the maximum $\Delta F / F$

454 within a 4-s after odor delivery. The ratio of the post/pre responses were calculated as the

455 maximum $\Delta F / F$ in an 8-s response window after odor delivery. In experiments with RNAi, flies

456 expressing GRAB-ACh, a UAS-RNAi line, and tub-Gal80 ${ }^{\text {ts }}$ were constructed; flies were raised at

$45718^{\circ} \mathrm{C}$ until eclosion, flies were transferred to $32^{\circ} \mathrm{C} 4-10$ days prior to the experiment.

458 Experiments were carried out at room temperature $\left(23^{\circ} \mathrm{C}\right)$ for $\mathrm{ACh}$ imaging/conditioning. For

$459 \mathrm{Ca}^{2+}$ imaging experiments, GCaMP6f was expressed in the MBONs using the R12G04

460 ( $\gamma 1$ pedc), MB077b $\left(\gamma 2 \alpha^{\prime} 1\right)$, MB083c $(\gamma 3)$ and VT014702 $\left(\gamma 5 \beta^{\prime 2}\right)$ Gal4 drivers. Experiments were

461 carried out same as ACh imaging, except presenting a 3s odor delivery.

462 Appetitive conditioning and imaging. Appetitive conditioning was carried out as previously

463 described (Louis et al., 2018). One odor (the CS+) was presented in conjunction with a paired

464 sucrose (1M, containing green food coloring) unconditioned stimulus (US), and a second odor

465 (the CS-) was presented 30-s later. Both the CS+ and CS- odors were presented during

466 conditioning experiments. In odor-only control cohorts, the sucrose US was omitted. During

467 training, each odor (and the US) was presented continuously for $30 \mathrm{~s}$ for $\mathrm{Ca}^{2+}$ imaging

468 experiments. Six 1-s odor pulses were presented during conditioning over a 30-s period, with a

469 5-s inter-pulse interval, to prevent desensitization of the reporter. Pre/post odor-evoked

470 responses were imaged prior to and after the imaging protocol, using a 3-s ( $\mathrm{Ca}^{2+}$ imaging) or 1-s

471 (ACh imaging) odor pulse. During odor-evoked response imaging, proboscis extension was

472 blocked utilizing a thin metal loop attached to a custom motorized micromanipulator. Flies were

473 starved for a period of $18-24 \mathrm{hrs}$ prior to conditioning. During conditioning, the proboscis was

474 released, and the flies were presented sucrose through a metal pipette fed by a syringe pump

475 controlled via a micro-controller (Arduino). To assess feeding, flies were monitored using a 
476 digital microscope (Vividia); sucrose ingestion was visually confirmed by the presence of green

477 food coloring in the abdomen.

478 Aversive conditioning and imaging. Flies were mounted in an aversive conditioning chamber

479 such that the brain could be imaged while odors were delivered to the antennae and electric

480 shocks delivered to the legs via a shock grid below the fly. Conditioning was carried out by

481 pairing a CS+ odor with electric shocks as follows: 6x 1-s odor pulses, with a 5-s inter-pulse

482 interval, paired with $6 \times 90-\mathrm{V}$ electric shocks, followed 30 s later by presentation of $6 \times 1$-s pulses

483 of the CS- odor with 5s inter-pulse interval. Pre- and post-conditioning odor-evoked responses

484 were imaged using a 1-s odor pulse. In each animal, either the CS+ or CS- odor was tested

485 pre- and post-conditioning.

486 Behavioral appetitive conditioning. Adult flies, 2-5 day old, were trained under dim red

487 light at $75 \%$ relative humidity. Appetitive conditioning experiments were performed in animals

488 starved $16-20$ h. Groups of $\sim 60$ flies were exposed for 2 min to an odor (the CS-), followed by

$48930 \mathrm{~s}$ of air and 2 min of another odor, the (the CS+), paired with a $1 \mathrm{M}$ sucrose solution dried on

490 filter paper, at $32^{\circ} \mathrm{C}$ for Shibire ${ }^{\text {ts }}$ blockade. The odor pairs were ethyl butyrate and isoamyl

491 acetate, adjusted so that naive flies equally avoided the two odors $(0.05-0.1 \%)$. Memory was

492 tested by inserting the trained flies into a T-maze, in which they chose between an arm

493 containing the CS+ odor and an arm containing the CS- odor. Flies were allowed to distribute

494 for a 2 min choice period. The Performance Index (P.I.), calculated as (flies in the CS- arm)-

495 (flies in the CS+ arm)/(total flies in both arms).

496 Immunohistochemistry. 5-7 days old adult flies were dissected in 1\% paraformaldehyde in S2

497 medium, and processed according to a published protocol (Jenett et al., 2012). Brains and

498 were incubated with the primary antibodies for 3 hours at room temperature and with the

499 secondary antibodies for 4 days at $4^{\circ} \mathrm{C}$. Incubations were performed in blocking serum ( $3 \%$ 
normal goat serum). Labeled brains were mounted in Vectashield media. Antibodies used were

501 rabbit anti-GFP (1:1000, Invitrogen), mouse anti-brp (nc82) (1:50, DSHB), mouse anti-

502 neuroglian (1:50,DSHB), goat anti-rabbit IgG and goat anti-mouse IgG (1:800, Alexa 488 or

503 Alexa 633 respectively, Invitrogen). Images were obtained using Leica TCS SP8 confocal

504 microscope.

505 Quantification and Statistical Analysis. Data were compared with ANOVA/Sidak

506 (parametric) or Kruskal-Wallis/Bonferroni (nonparametric) tests. Box plots show graph the 507 median as a line, the $1^{\text {st }}$ and $3^{\text {rd }}$ quartile enclosed in the box, and whiskers extending from the $50810^{\text {th }}$ to the $90^{\text {th }}$ percentile.

\section{Acknowledgments}

511 The authors thank Krystyna Keleman for fly stocks, and Brock Grill for helpful discussions.

512 Stocks obtained from the Bloomington Drosophila Stock Center (NIH P40OD018537) were used

513 in this study. We thank Yuexuan Li for the help in the development of the GRAB-ACh sensor.

514 Research support was provided by NIH R00MH092294, R01 NS097237, the Whitehall

515 Foundation (S.M.T.), NIH R35NS097224 (R.L.D.), the Beijing Municipal Science \& Technology

516 Commission Z181100001318002 (Y.L.), the Beijing Brain Initiative of Beijing Municipal Science

517 \& Technology Commission Z181100001518004 (Y.L.), Guangdong Grant "Key Technologies for

518 Treatment of Brain Disorders" 2018B030332001 (Y.L.), the General Program of National Natural

519 Science Foundation of China projects 31671118, 31871087, and 31925017) (Y.L.), the NIH

520 BRAIN Initiative NS103558 (Y.L.), grants from the Peking-Tsinghua Center for Life Sciences

521 (Y.L.) and the State Key Laboratory of Membrane Biology at Peking University School of Life

522 Sciences (Y. L.). 
Competing Interests

The authors declare no competing financial interests.

$\underline{\text { References }}$

Aso, Y., Hattori, D., Yu, Y., Johnston, R.M., Iyer, N.A., Ngo, T.T., Dionne, H., Abbott, L., Axel, R., Tanimoto, H., and Rubin, G.M. (2014a). The neuronal architecture of the mushroom body provides a logic for associative learning. Elife 3.

Aso, Y., Sitaraman, D., Ichinose, T., Kaun, K.R., Vogt, K., Belliart-Guerin, G., Placais, P.Y., Robie, A.A., Yamagata, N., Schnaitmann, C., et al. (2014b). Mushroom body output neurons encode valence and guide memory-based action selection in Drosophila. Elife 3. Barnstedt, O., Owald, D., Felsenberg, J., Brain, R., Moszynski, J.P., Talbot, C.B., Perrat, P.N., and Waddell, S. (2016). Memory-Relevant Mushroom Body Output Synapses Are Cholinergic. Neuron 89, 1237-1247.

Berry, J.A., Phan, A., and Davis, R.L. (2018). Dopamine Neurons Mediate Learning and Forgetting through Bidirectional Modulation of a Memory Trace. Cell Rep 25, 651-662 e655. Boto, T., Louis, T., Jindachomthong, K., Jalink, K., and Tomchik, S.M. (2014). Dopaminergic Modulation of cAMP Drives Nonlinear Plasticity across the Drosophila Mushroom Body Lobes. Curr Biol 24, 822-831.

Boto, T., Stahl, A., Zhang, X., Louis, T., and Tomchik, S.M. (2019). Independent Contributions of Discrete Dopaminergic Circuits to Cellular Plasticity, Memory Strength, and Valence in Drosophila. Cell Rep 27, 2014-2021 e2012.

Cervantes-Sandoval, I., Phan, A., Chakraborty, M., and Davis, R.L. (2017). Reciprocal synapses between mushroom body and dopamine neurons form a positive feedback loop required for learning. Elife 6 .

Cohn, R., Morantte, I., and Ruta, V. (2015). Coordinated and Compartmentalized Neuromodulation Shapes Sensory Processing in Drosophila. Cell 163, 1742-1755. Crittenden, J.R., Skoulakis, E.M., Han, K.A., Kalderon, D., and Davis, R.L. (1998). Tripartite mushroom body architecture revealed by antigenic markers. Learn Mem 5, 38-51. Dietzl, G., Chen, D., Schnorrer, F., Su, K.C., Barinova, Y., Fellner, M., Gasser, B., Kinsey, K., Oppel, S., Scheiblauer, S., et al. (2007). A genome-wide transgenic RNAi library for conditional gene inactivation in Drosophila. Nature 448, 151-156.

Frank, C.A., Kennedy, M.J., Goold, C.P., Marek, K.W., and Davis, G.W. (2006). Mechanisms underlying the rapid induction and sustained expression of synaptic homeostasis. Neuron 52, 663-677.

Gervasi, N., Tchenio, P., and Preat, T. (2010). PKA dynamics in a Drosophila learning center: coincidence detection by rutabaga adenylyl cyclase and spatial regulation by dunce phosphodiesterase. Neuron 65, 516-529.

Gratz, S.J., Goel, P., Bruckner, J.J., Hernandez, R.X., Khateeb, K., Macleod, G.T., Dickman, D., and O'Connor-Giles, K.M. (2019). Endogenous Tagging Reveals Differential Regulation of $\mathrm{Ca}(2+)$ Channels at Single Active Zones during Presynaptic Homeostatic Potentiation and Depression. J Neurosci 39, 2416-2429.

Handler, A., Graham, T.G.W., Cohn, R., Morantte, I., Siliciano, A.F., Zeng, J., Li, Y., and Ruta, V. (2019). Distinct Dopamine Receptor Pathways Underlie the Temporal Sensitivity of Associative Learning. Cell 178, 60-75 e19. 
569

570

571

572

573

574

575

576

577

578

579

580

581

582

583

584

585

586

587

588

589

590

591

592

593

594

595

596

597

598

599

600

601

602

603

604

605

606

607

608

609

610

611

612

613

614

615

616

617

618

Hendricks, M., Ha, H., Maffey, N., and Zhang, Y. (2012). Compartmentalized calcium dynamics in a C. elegans interneuron encode head movement. Nature 487, 99-103.

Hige, T., Aso, Y., Modi, M.N., Rubin, G.M., and Turner, G.C. (2015a). Heterosynaptic Plasticity Underlies Aversive Olfactory Learning in Drosophila. Neuron 88, 985-998.

Hige, T., Aso, Y., Rubin, G.M., and Turner, G.C. (2015b). Plasticity-driven individualization of olfactory coding in mushroom body output neurons. Nature 526, 258-262.

Ichinose, T., Aso, Y., Yamagata, N., Abe, A., Rubin, G.M., and Tanimoto, H. (2015). Reward signal in a recurrent circuit drives appetitive long-term memory formation. Elife 4, e10719.

Inchauspe, C.G., Martini, F.J., Forsythe, I.D., and Uchitel, O.D. (2004). Functional

compensation of $P / Q$ by $N$-type channels blocks short-term plasticity at the calyx of Held presynaptic terminal. J Neurosci 24, 10379-10383.

Ishikawa, T., Kaneko, M., Shin, H.S., and Takahashi, T. (2005). Presynaptic N-type and P/Qtype Ca2+ channels mediating synaptic transmission at the calyx of Held of mice. J Physiol 568, 199-209.

James, T.D., Zwiefelhofer, D.J., and Frank, C.A. (2019). Maintenance of homeostatic plasticity at the Drosophila neuromuscular synapse requires continuous IP3-directed signaling. Elife 8. Jenett, A., Rubin, G.M., Ngo, T.T., Shepherd, D., Murphy, C., Dionne, H., Pfeiffer, B.D., Cavallaro, A., Hall, D., Jeter, J., et al. (2012). A GAL4-driver line resource for Drosophila neurobiology. Cell Rep 2, 991-1001.

Jing, M., Li, Y., Zeng, J., Huang, P., Skirzewski, M., Kljakic, O., Peng, W., Qian, T., Tan, K., and Wu, R. (2019). An optimized acetylcholine sensor for monitoring in vivo cholinergic activity. bioRxiv, 861690.

Jing, M., Zhang, P., Wang, G., Feng, J., Mesik, L., Zeng, J., Jiang, H., Wang, S., Looby, J.C., Guagliardo, N.A., et al. (2018). A genetically encoded fluorescent acetylcholine indicator for in vitro and in vivo studies. Nat Biotechnol 36, 726-737.

Kim, Y.C., Lee, H.G., and Han, K.A. (2007). D1 dopamine receptor dDA1 is required in the mushroom body neurons for aversive and appetitive learning in Drosophila. J Neurosci 27 , 7640-7647.

Konig, C., Khalili, A., Ganesan, M., Nishu, A.P., Garza, A.P., Niewalda, T., Gerber, B., Aso, Y., and Yarali, A. (2018). Reinforcement signaling of punishment versus relief in fruit flies. Learn Mem 25, 247-257.

Liu, C., Placais, P.Y., Yamagata, N., Pfeiffer, B.D., Aso, Y., Friedrich, A.B., Siwanowicz, I., Rubin, G.M., Preat, T., and Tanimoto, H. (2012). A subset of dopamine neurons signals reward for odour memory in Drosophila. Nature 488, 512-516.

Louis, T., Stahl, A., Boto, T., and Tomchik, S.M. (2018). Cyclic AMP-dependent plasticity underlies rapid changes in odor coding associated with reward learning. Proc Natl Acad Sci U S A 115, E448-E457.

Lubbert, M., Goral, R.O., Keine, C., Thomas, C., Guerrero-Given, D., Putzke, T., Satterfield, R., Kamasawa, N., and Young, S.M., Jr. (2019). CaV2.1 alpha1 Subunit Expression Regulates Presynaptic CaV2.1 Abundance and Synaptic Strength at a Central Synapse. Neuron 101, 260273 e266.

Mao, Z., and Davis, R.L. (2009). Eight different types of dopaminergic neurons innervate the Drosophila mushroom body neuropil: anatomical and physiological heterogeneity. Front Neural Circuits 3, 5.

McGuire, S.E., Le, P.T., and Davis, R.L. (2001). The role of Drosophila mushroom body signaling in olfactory memory. Science 293, 1330-1333.

McGuire, S.E., Le, P.T., Osborn, A.J., Matsumoto, K., and Davis, R.L. (2003). Spatiotemporal rescue of memory dysfunction in Drosophila. Science 302, 1765-1768.

Modi, M.N., Shuai, Y., and Turner, G.C. (2020). The Drosophila Mushroom Body: From Architecture to Algorithm in a Learning Circuit. Annu Rev Neurosci 43, 465-484. 
619

620

621

622

623

624

625

626

627

628

629

630

631

632

633

634

635

636

637

638

639

640

641

642

643

644

645

646

647

648

649

650

651

652

653

654

655

656

657

658

659

660

661

662

663

664

665

666

667

668

669

Muller, M., and Davis, G.W. (2012). Transsynaptic control of presynaptic Ca(2)(+) influx achieves homeostatic potentiation of neurotransmitter release. Curr Biol 22, 1102-1108. Nanou, E., Scheuer, T., and Catterall, W.A. (2016). Calcium sensor regulation of the CaV2.1 $\mathrm{Ca} 2+$ channel contributes to long-term potentiation and spatial learning. Proc Natl Acad Sci U S A $113,13209-13214$.

Owald, D., Felsenberg, J., Talbot, C.B., Das, G., Perisse, E., Huetteroth, W., and Waddell, S. (2015). Activity of defined mushroom body output neurons underlies learned olfactory behavior in Drosophila. Neuron 86, 417-427.

Perisse, E., Owald, D., Barnstedt, O., Talbot, C.B., Huetteroth, W., and Waddell, S. (2016). Aversive Learning and Appetitive Motivation Toggle Feed-Forward Inhibition in the Drosophila Mushroom Body. Neuron 90, 1086-1099.

Perkins, L.A., Holderbaum, L., Tao, R., Hu, Y., Sopko, R., McCall, K., Yang-Zhou, D., Flockhart, I., Binari, R., Shim, H.S., et al. (2015). The Transgenic RNAi Project at Harvard Medical School: Resources and Validation. Genetics 201, 843-852.

Pfeiffer, B.D., Ngo, T.T., Hibbard, K.L., Murphy, C., Jenett, A., Truman, J.W., and Rubin, G.M. (2010). Refinement of tools for targeted gene expression in Drosophila. Genetics 186, 735-755. Placais, P.Y., Trannoy, S., Friedrich, A.B., Tanimoto, H., and Preat, T. (2013). Two pairs of mushroom body efferent neurons are required for appetitive long-term memory retrieval in Drosophila. Cell Rep 5, 769-780.

Rowan, M.J., DelCanto, G., Yu, J.J., Kamasawa, N., and Christie, J.M. (2016). Synapse-Level Determination of Action Potential Duration by $\mathrm{K}(+)$ Channel Clustering in Axons. Neuron 91, 370-383.

Sayin, S., De Backer, J.F., Siju, K.P., Wosniack, M.E., Lewis, L.P., Frisch, L.M., Gansen, B., Schlegel, P., Edmondson-Stait, A., Sharifi, N., et al. (2019). A Neural Circuit Arbitrates between Persistence and Withdrawal in Hungry Drosophila. Neuron 104, 544-558 e546.

Scaplen, K.M., Talay, M., Fisher, J.D., Cohn, R., Sorkac, A., Aso, Y., Barnea, G., and Kaun, K.R. (2021). Transsynaptic mapping of Drosophila mushroom body output neurons. Elife 10. Schroll, C., Riemensperger, T., Bucher, D., Ehmer, J., Voller, T., Erbguth, K., Gerber, B., Hendel, T., Nagel, G., Buchner, E., and Fiala, A. (2006). Light-induced activation of distinct modulatory neurons triggers appetitive or aversive learning in Drosophila larvae. Curr Biol 16, 1741-1747.

Schwaerzel, M., Monastirioti, M., Scholz, H., Friggi-Grelin, F., Birman, S., and Heisenberg, M. (2003). Dopamine and octopamine differentiate between aversive and appetitive olfactory memories in Drosophila. J Neurosci 23, 10495-10502.

Sejourne, J., Placais, P.Y., Aso, Y., Siwanowicz, I., Trannoy, S., Thoma, V., Tedjakumala, S.R., Rubin, G.M., Tchenio, P., Ito, K., et al. (2011). Mushroom body efferent neurons responsible for aversive olfactory memory retrieval in Drosophila. Nat Neurosci 14, 903-910.

Takemura, S.Y., Aso, Y., Hige, T., Wong, A., Lu, Z., Xu, C.S., Rivlin, P.K., Hess, H., Zhao, T., Parag, T., et al. (2017). A connectome of a learning and memory center in the adult Drosophila brain. Elife 6.

Tanaka, N.K., Tanimoto, H., and Ito, K. (2008). Neuronal assemblies of the Drosophila mushroom body. J Comp Neurol 508, 711-755.

Tanimoto, H., Heisenberg, M., and Gerber, B. (2004). Experimental psychology: event timing turns punishment to reward. Nature 430, 983.

Taufiq, A.M., Fujii, S., Yamazaki, Y., Sasaki, H., Kaneko, K., Li, J., Kato, H., and Mikoshiba, K. (2005). Involvement of IP3 receptors in LTP and LTD induction in guinea pig hippocampal CA1 neurons. Learn Mem 12, 594-600.

Tomchik, S.M., and Davis, R.L. (2009). Dynamics of learning-related cAMP signaling and stimulus integration in the Drosophila olfactory pathway. Neuron 64, 510-521.

Tully, T., and Quinn, W.G. (1985). Classical conditioning and retention in normal and mutant Drosophila melanogaster. J Comp Physiol A 157, 263-277. 
670 Xu, C.S., Januszewski, M., Lu, Z., Takemura, S.-y., Hayworth, K., Huang, G., Shinomiya, K., 671 Maitin-Shepard, J., Ackerman, D., and Berg, S. (2020). A Connectome of the Adult Drosophila 672 Central Brain. bioRxiv.

673 Yamagata, N., Hiroi, M., Kondo, S., Abe, A., and Tanimoto, H. (2016). Suppression of

674 Dopamine Neurons Mediates Reward. PLoS Biol 14, e1002586.

675 Yamagata, N., Ichinose, T., Aso, Y., Placais, P.Y., Friedrich, A.B., Sima, R.J., Preat, T., Rubin, 676 G.M., and Tanimoto, H. (2015). Distinct dopamine neurons mediate reward signals for short677 and long-term memories. Proc Natl Acad Sci U S A 112, 578-583.

678 Zamponi, G.W., and Currie, K.P. (2013). Regulation of $\mathrm{Ca}(\mathrm{V}) 2$ calcium channels by $\mathrm{G}$ protein 679 coupled receptors. Biochim Biophys Acta 1828, 1629-1643.

680 Zars, T., Fischer, M., Schulz, R., and Heisenberg, M. (2000). Localization of a short-term 681 memory in Drosophila. Science 288, 672-675.

682 Zhang, S., and Roman, G. (2013). Presynaptic inhibition of gamma lobe neurons is required for 683 olfactory learning in Drosophila. Curr Biol 23, 2519-2527.

684 Zhang, X., Noyes, N.C., Zeng, J., Li, Y., and Davis, R.L. (2019). Aversive Training Induces Both 685 Presynaptic and Postsynaptic Suppression in Drosophila. J Neurosci 39, 9164-9172.

686 Zhao, X., Lenek, D., Dag, U., Dickson, B.J., and Keleman, K. (2018). Persistent activity in a 687 recurrent circuit underlies courtship memory in Drosophila. Elife 7. 
bioRxiv preprint doi: https://doi.org/10.1101/2021.06.08.447536; this version posted June 10, 2021. The copyright holder for this preprint (which was not certified by peer review) is the author/funder, who has granted bioRxiv a license to display the preprint in perpetuity. It is made available under aCC-BY 4.0 International license.
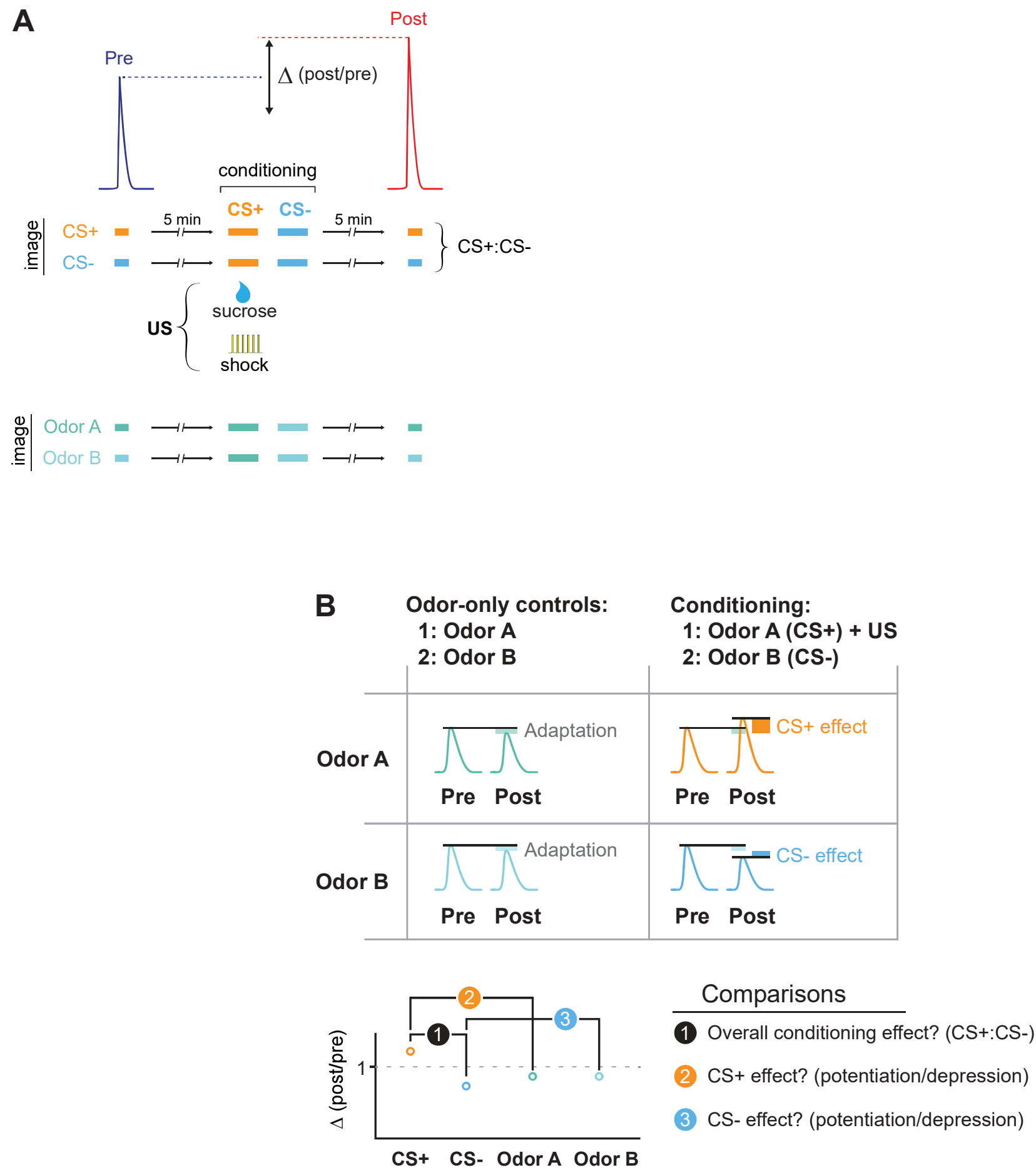

CS+ CS- Odor A Odor B

Figure S1, related to Figure 1. The conditioning and imaging assay and data analysis. (A) Flies were conditioned by pairing an odor (the CS+) with a US (electric shock or sucrose reward), and a second odor (the CS-) was presented afterward. Odor-evoked GACh or GCaMP responses were imaged in the MB and compared before (Pre) and after (Post) conditioning. Responses were compared to animals in which the same odors were presented, but no US presented (odor-only controls). To examine how conditioning (or odor-only presentation) changed the odor responses, the $\Delta$ (post/pre) was calculated for each treatment. (B) Two types of comparisons were made across conditions. First, we analyzed the CS+:CS- ratio, which mimics the putative comparison the animal makes when comparing the two odors at the choice point in a T-maze during memory retrieval. Second, we compared the CS+ and CS- to their respective odor-only controls in order to determine whether the responses were potentiated or depressed by conditioning. This comparison normalizes for any olfactory adaptation that is induced by the odor presentation during the training window. 

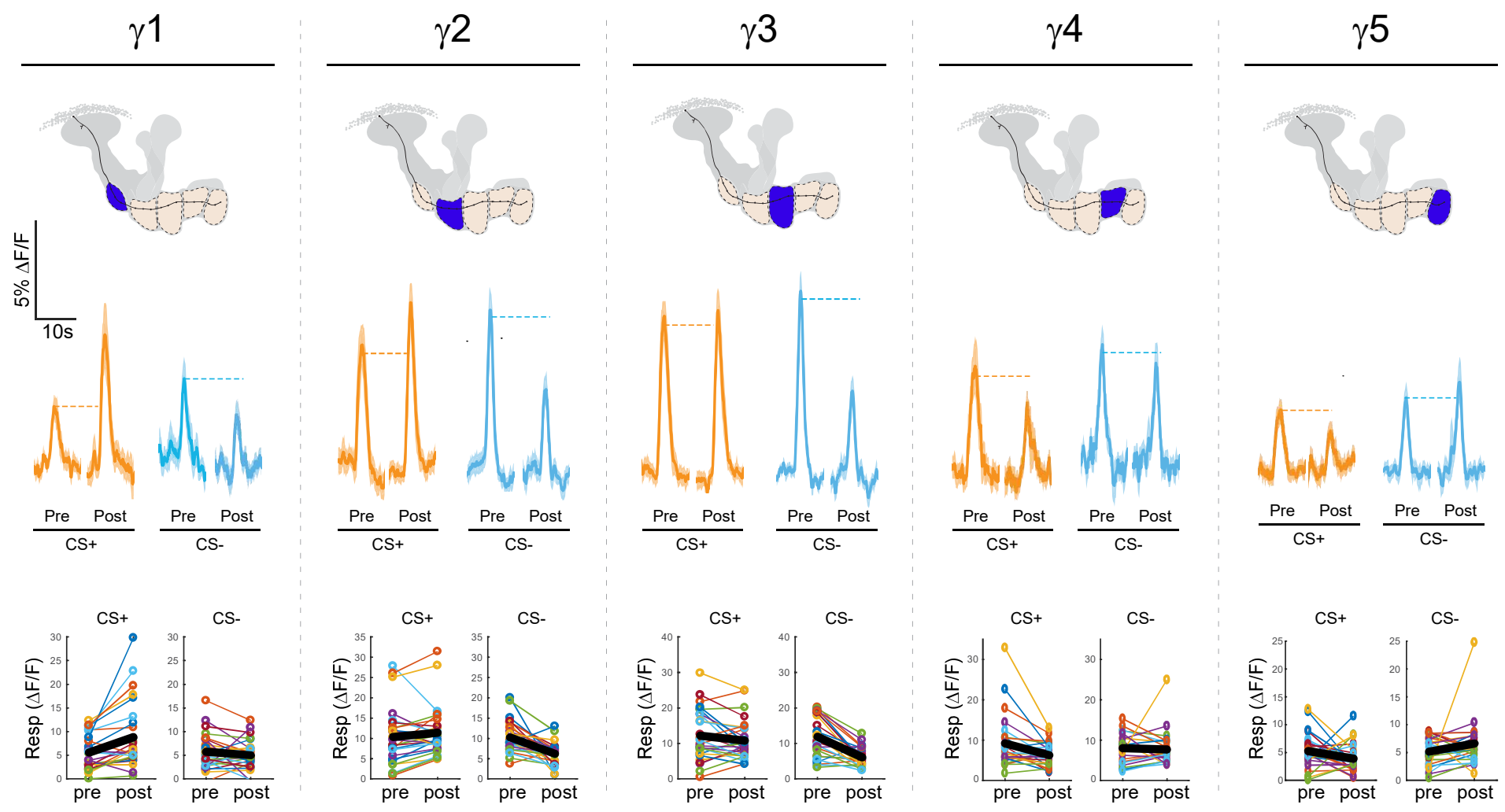

Figure S2, related to Figure 1. Effects of appetitive conditioning on GRAB-ACh responses across the $\gamma$ lobe compartments. The top row shows diagrams of the location of each compartment within the mushroom body. The second row shows time series traces pre- and post-conditioning for the CS+ (ethyl butyrate [EB]) and CS(isoamyl acetate [IA]). The third row shows quantification of the peak pre- and post-conditioning responses for each animal $(n=27)$. The thick black line represents the mean. 

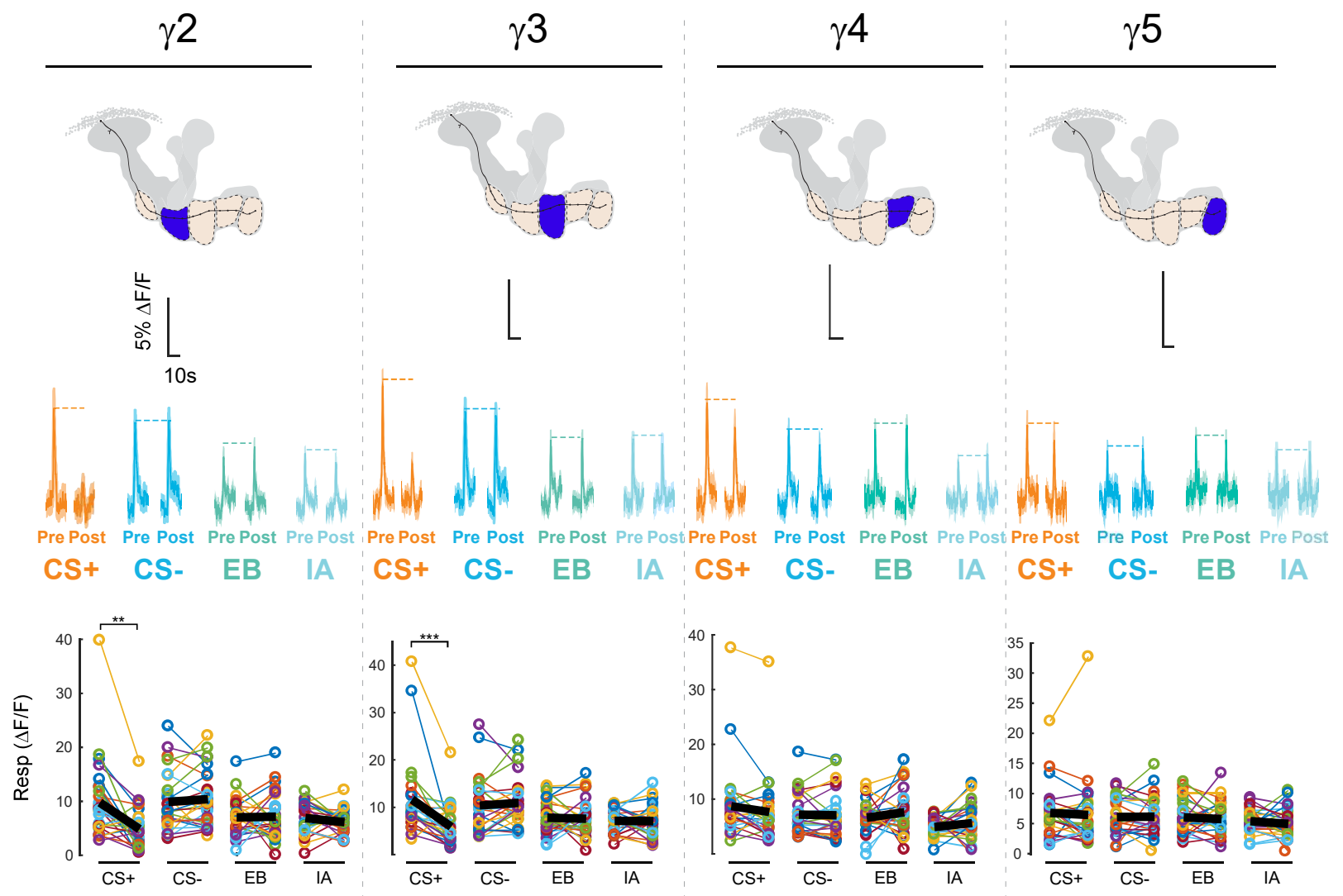

Figure S3, related to Figure 2. Effects of averisve conditioning on GRAB-ACh responses across the $\gamma$ lobe compartments. The top row shows diagrams of the location of each compartment within the mushroom body. The second row shows time series traces pre- and post-conditioning for the CS+ (ethyl butyrate [EB]) and CS(isoamyl acetate $[\mathrm{IA}]$ ) and odor only controls. The third row shows quantification of the peak pre- and post-conditioning responses for each animal $(n=27){ }^{*} p<0.05,{ }^{*} p<0.005,{ }^{* * *} p<0.0005 n=12$ (Wilcoxon rank-sum test) The thick black line represents the mean. 

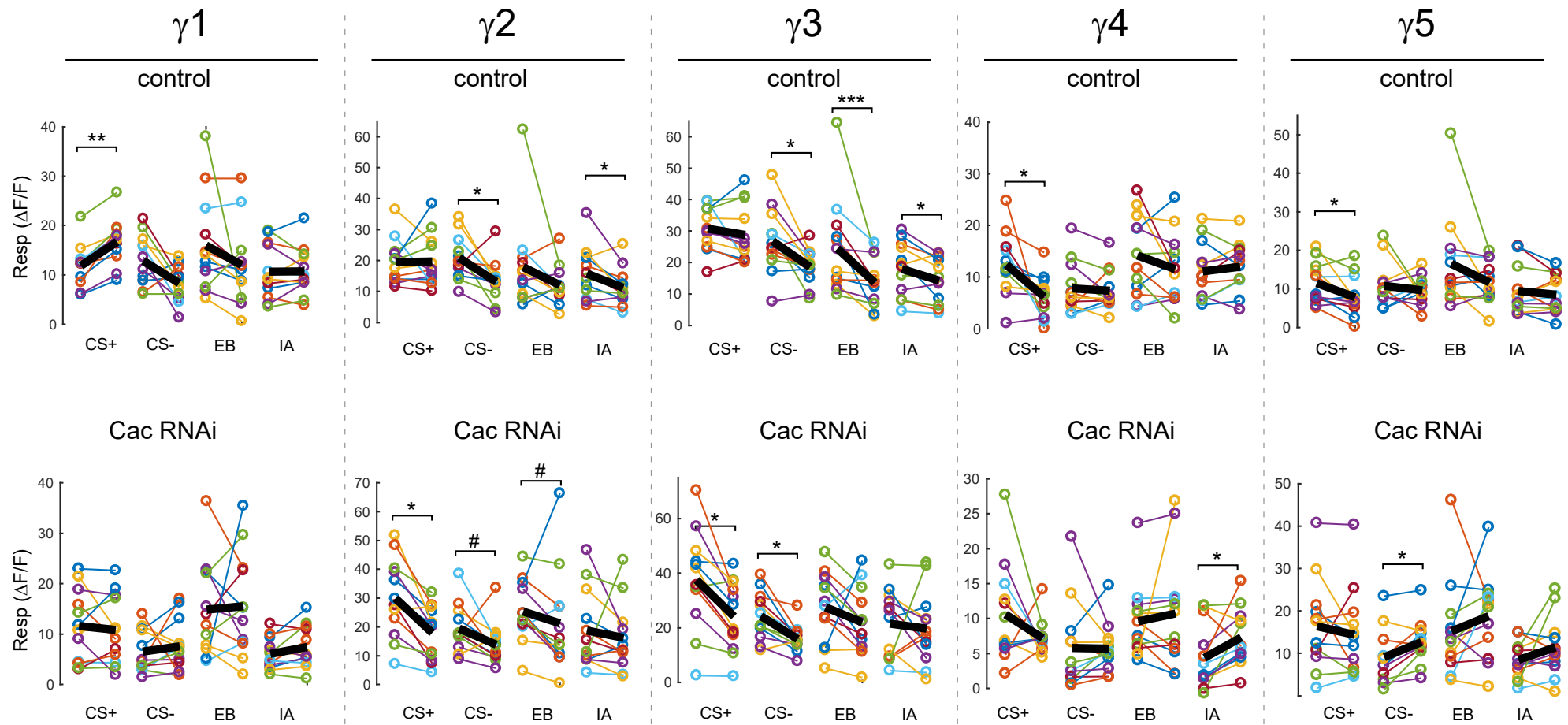

Figure S4, related to Figure 3. Effects of appetitive conditioning on GRAB-ACh responses across the $\gamma$ lobe compartments using GRAB-ACh with control and cacophony knockdown flies. The top row shows time series traces pre- and post-conditioning for the CS+ (ethyl butyrate [EB]) and CS- (isoamyl acetate [IA]) of control flies. The quantification of the peak pre- and post-conditioning responses for each animal $(n=12)$ of control flies. ${ }^{*} p<0.05,{ }^{*} p<0.005,{ }^{* * *} p<0.0005, \# p<0.07, n=12$ (Wilcoxon rank-sum test). The second row shows time series traces pre- and post-conditioning Cac knockdowns. 
bioRxiv preprint doi: https://doi.org/10.1101/2021.06.08.447536; this version posted June 10, 2021. The copyright holder for this preprint (which was not certified by peer review) is the author/funder, who has granted bioRxiv a license to display the preprint in perpetuity. It is made $\gamma 1$ $\gamma 2$ available under aCC-BY $4.93^{\text {ternational license. }}$

$\gamma 5$
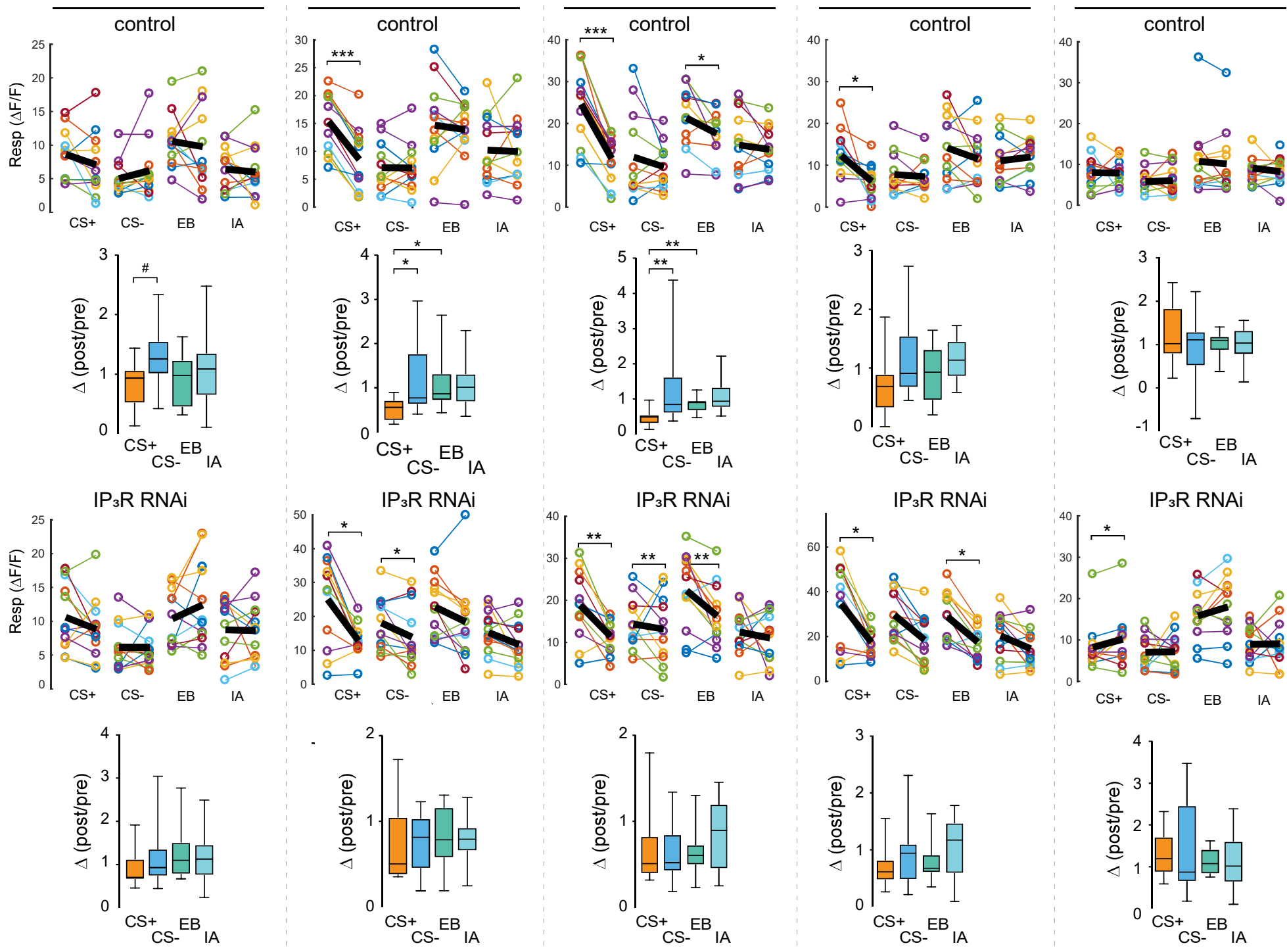

Cac RNAi

Cac RNAi

Cac RNAi
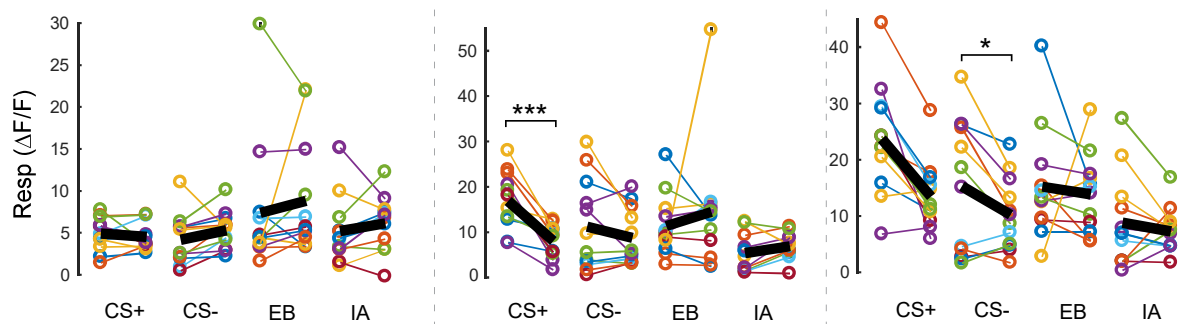

Cac RNAi

Cac RNAi
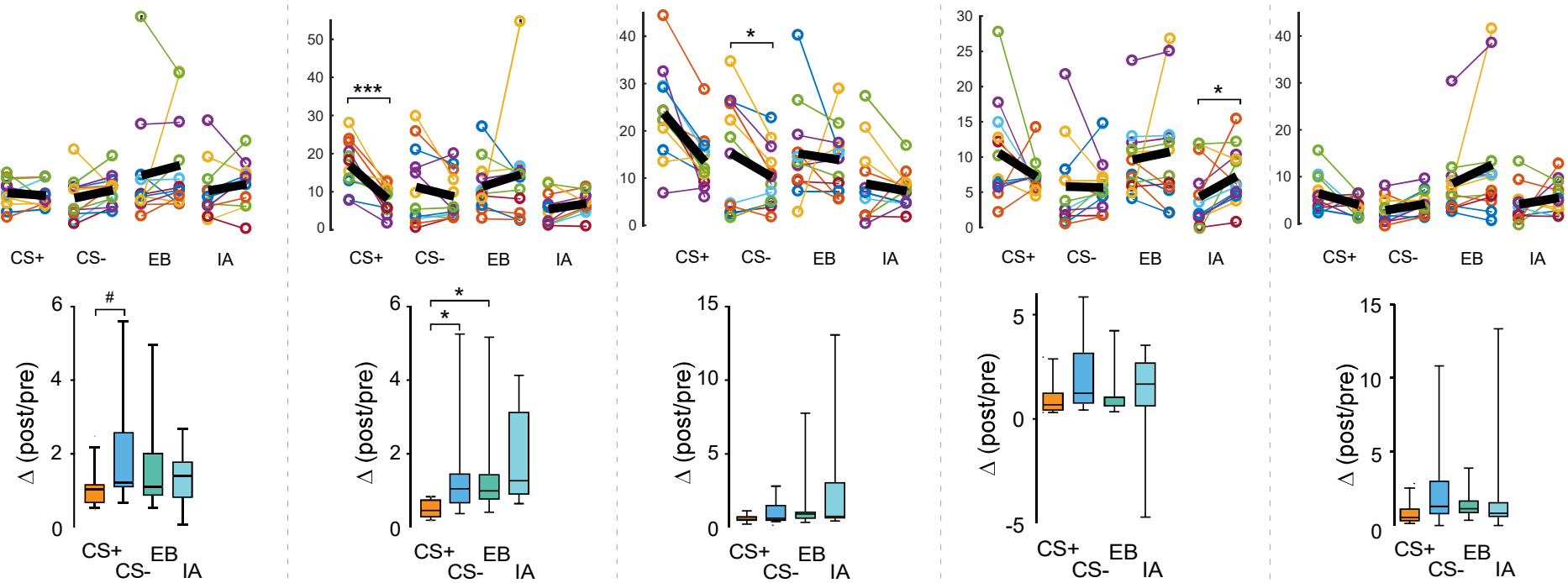

Figure S5, related to Figure 4. Effects of aversive conditioning on GRAB-ACh responses across the $\gamma$ lobe compartments using GRAB-ACh with control, cacophony and $\mathrm{IP}_{3} \mathrm{R}$ knockdown flies. For all genotypes sample sizes, $\mathrm{n}=12$ with statistical analysis (Wilcoxon rank-sum test) ${ }^{*} p<0.05,{ }^{*} p<0.005,{ }^{* * *} p<0.0005$ for time series traces. For comparisons of CS+, CS-, and odor-only control responses (Kruskal-Wallis/Bonferroni) \#p<0.03, ${ }^{*} p<0.01,{ }^{* *} p<0.001,{ }^{* *} p<0.0001$. The top row shows time series traces pre- and post-conditioning for the CS+ (ethyl butyrate [EB]) and CS- (isoamyl acetate [IA]) and odor only, and the thick black line represents the mean. The second row shows comparisons of the CS+, CS-, and odor-only controls. The third row shows time series traces pre-post conditioning for $\mathrm{IP}_{3} \mathrm{R}$ knockdowns. The fourth row shows comparisons between the four treatments of IP3R knockdowns. The fifth row shows time series traces pre-post conditioning for Cac knockdowns. The final row shows comparisons between the four treatments of Cac knockdowns. 

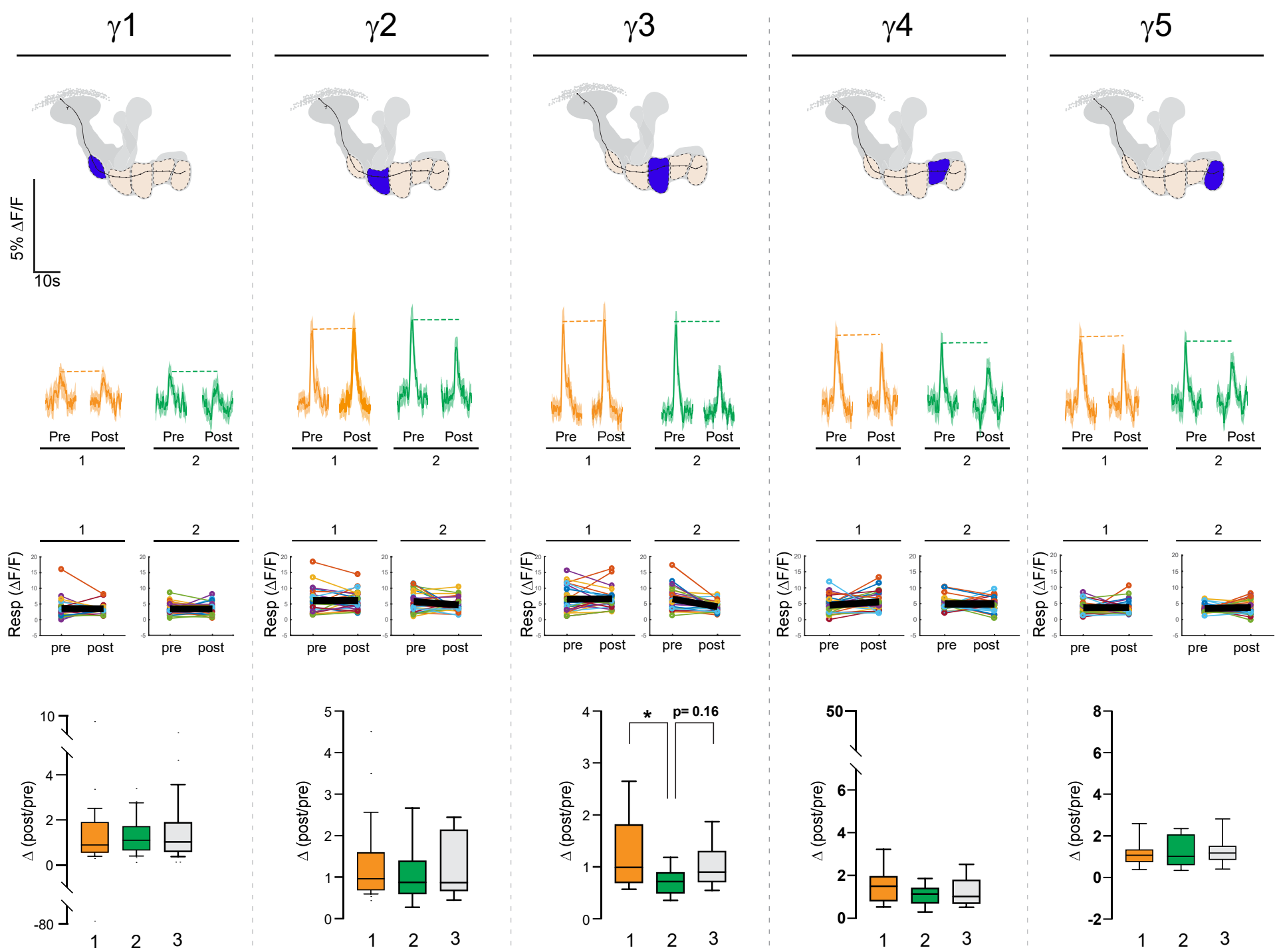

Figure S6, related to Figure 6. Effects of appetitive conditioning on GRAB-ACh responses across the $\gamma$ lobe in the absence of either CS+ (1) or CS- (2). The top row shows diagrams of the location of each compartment within the mushroom body. The second row shows time series traces pre- and post-conditioning for paired, unpaired, and odor-only conditioning. The third row shows quantification of the peak pre- and post-conditioning responses for each animal $(n=27)$. The thick black line represents the mean. The bottom row shows comparisons of the CS+, CS-, and odor-only controls (EB and IA). ${ }^{*} p<0.01,{ }^{* *} p<0.001$, ${ }^{* * *} p<0.0001 ; n=27$ (Kruskal-Wallis/Bonferroni). 